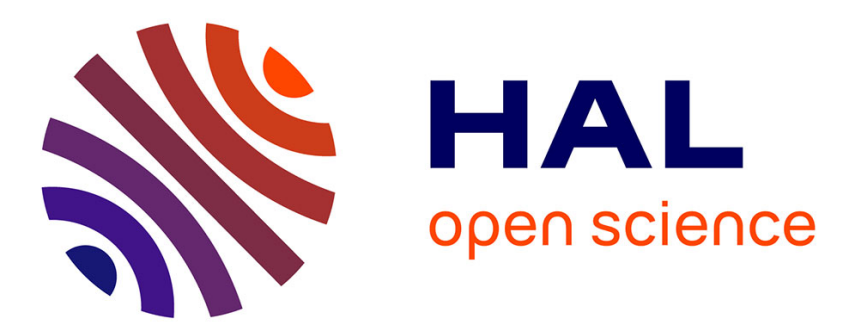

\title{
Absorbing boundary conditions for linear gravity waves
} Kebir Dgaygui, Patrick Joly

\section{To cite this version:}

Kebir Dgaygui, Patrick Joly. Absorbing boundary conditions for linear gravity waves. [Research Report] RR-1600, INRIA. 1992. inria-00074960

\section{HAL Id: inria-00074960 https://hal.inria.fr/inria-00074960}

Submitted on 24 May 2006

HAL is a multi-disciplinary open access archive for the deposit and dissemination of scientific research documents, whether they are published or not. The documents may come from teaching and research institutions in France or abroad, or from public or private research centers.
L'archive ouverte pluridisciplinaire HAL, est destinée au dépôt et à la diffusion de documents scientifiques de niveau recherche, publiés ou non, émanant des établissements d'enseignement et de recherche français ou étrangers, des laboratoires publics ou privés. 


\title{
ABSORBING BOUNDARY CONDITIONS FOR LINEAR GRAVITY WAVES CONDITIONS ABSORBANTES POUR LES ONDES DE GRAVITE EN REGIME LINEAIRE
}

Kebir DGAYGUI and Patrick JOLY

INRIA B.P. 105,78153 Le Chesnay , France

\begin{abstract}
:
In this article,we construct,analyze and implement a family of absorbing boundary conditions for linear gravity waves in dimension 2 . The main difficulty consists in taking into account the dispersive nature of these waves.
\end{abstract}

\section{Résumé :}

Dans cet article,nous construisons,analysons et implémentons une famille de conditions aux limites absorbantes pour les ondes de gravité linéaires en dimension 2, qui modélisent la propagation de la houle.La principale difficulté consiste à tenir compte de la nature dispersive de ces ondes.

Key words :

Gravity waves, dispersive waves,absorbing boudary conditions,rational approximation, stability analysis.

Mots clés :

Ondes de gravité,ondes dispersives,conditions aux limites absorbantes, approximation rationnelle, analyse de stabilité. 


\section{Introduction - Position of the problem}

Absorbing boundary conditions for wave propagation models in infinite domains of propagation are an essential tool for the numerical approximation of such physical phenomena. Such boundary conditions have been developed ten years ago for acoustic wave and elastic wave propagation in the whole space by B. Engquist and A. Majda. Since this date several research works have been devoted to the generalization of their conditions to different domains of the physics as acoustic waves $([4],[5],[12])$, elastic waves $([3],[8],[13])$, electromagnetism $([7],[22])$, diffusion phenomena $([21])$, fluid dynamics $([17],[18],[26])$.

Linear water waves in the ocean in dimension $N+1(N=1$ or 2$)$ obey to a model which has the following particular properties :

- the domain of propagation is bounded in one direction and unbounded in $N$ directions. It can be assimilated to a slab.

- the evolution problem, and thus the propagation phenomenon, is generated by the boundary condition on the free surface of the ocean and not by the partial differential equation itself.

In [15], Hagström proposed a general method for developing artificial boundary conditions for general evolution problems posed in a slab,in the case in which the evolution term is mainly contained in the partial differential equation governing the solution in the interior of the domain of propagation. His method is based on a spectral decomposition of a differential operator in the transverse variables (with respect to the direction of the slab). We use here the same type of method for developing absorbing boundary conditions for the linear water waves model in dimension $2(N=1)$. We construct a general family of such boundary conditions that we analyze from both theoretical and computational points of view. The content of our paper is the following one.

In section 2 we recall the main mathematical properties of the water waves model. We compare these properties with the ones of the acoustic wave equation. We point out the dispersive character of harmonic waves associated to our model and the infinite velocity of propagation.

Section 3, which is the most important one, is devoted to the derivation of a transparent boundary condition (section 3.1), the heuristic construction of the absorbing boundary conditions (section 3.2), the theoretical analysis of the well posedness of the corresponding initial boundary value problem (section 3.3), the presentation of a numerical method for its approximation (section 3.4) and a discussion about different possible choices for the 
boundary conditions. As it is classical, the construction of the artificial boundary condition is based on a local (in space and time) approximation of the transparent boundary condition. The spectral representation for an appropriate transverse operator is used for the expression of this transparent boundary condition. We try to establish a parallel with the case of the acoustic wave equation. The main difference comes from the fact that we have to take into account the dispersive nature of the water waves. In the theoretical part, the main difficulty comes from the fact that we cannot apply directly the stability theory developed by Kreiss $([19],[20],[22])$ since we are not working with an hyperbolic system. The approach we generally adopt is a combination of the modal analysis (we use a Laplace transform with respect to time) and energy techniques.

Finally, we present in section 4 several numerical results which give the validation of our approach. In conclusion, we shall give some elements concerning the extension of the present work to the $3 \mathrm{D}$ problem, that we shall develop in a forthcoming paper.

Let us note that an alternative numerical method to treat infinite domains of propagation has been proposed by M. Lenoir and M. Verrière (see [26] and [31]). Their method is not based on the construction of absorbing boundary conditions. It rests upon a time semi-discretization of the evolution problem which leads to a sequence of stationnary linear problems at each time step. A decomposition problem leads to solve two different problems : one is solved quasi-exactly by an analytical method and the other is approximated via an integral representation boundary condition [31] or alternatively by the so-called localized finite element method [25]. Such an approach reveals rather precise but also rather costly from a computational point of view. We think that the use of our boundary condition reduces the computational cost of the simulation without affecting the accuracy of the results.

\section{Presentation of the model and description of its main mathematical properties}

In this paragraph, we shall work in $l R^{N+1}$, with $N=1$ or 2 . We shall denote by $(x, y)$ a point of $l R^{N+1}, x \in l R^{N}$ and $y \in l R$. The privileged direction $y$ corresponds physically to the vertical direction or depth. The time will be denoted by $t \in l R^{+}$.

The aim of the linearized water waves model is to describe the movement of the ocean, or more precisely the displacement of the material particles of the fluid which is identified to the ocean.

We shall be interested in this paper by finite depth oceans which means physically that we shall have essentially wave phenomena whose wave length is not too small with respect to the mean depth of the ocean. 
In order to derive the model of linear water waves (we refer the reader to [29], for instance, for further details), one has to do the following assumptions :

$$
\left\{\begin{array}{l}
\text { the fluid is perfect and incompressible, } \\
\text { the only exterior field is the gravity, } \\
\text { the flow is irrotational. }
\end{array}\right.
$$

We shall denote by $\Omega$ the volume occupied by the fluid and assume that its boundary $(\Gamma)$ can be splitted in two parts :

$$
(\Gamma)=\left(\Gamma_{B}\right) \cup\left(\Gamma_{F}\right)
$$

where $\left(\Gamma_{B}\right)$ denotes the bottom of the ocean and $\left(\Gamma_{F}\right)$ denotes the free boundary. Under the hypotheses of small displacements, it is assumed that $\Omega$ and $\Gamma$ are independent on time and that $\left(\Gamma_{F}\right)$ coincides with a part of the plane $y=0$.

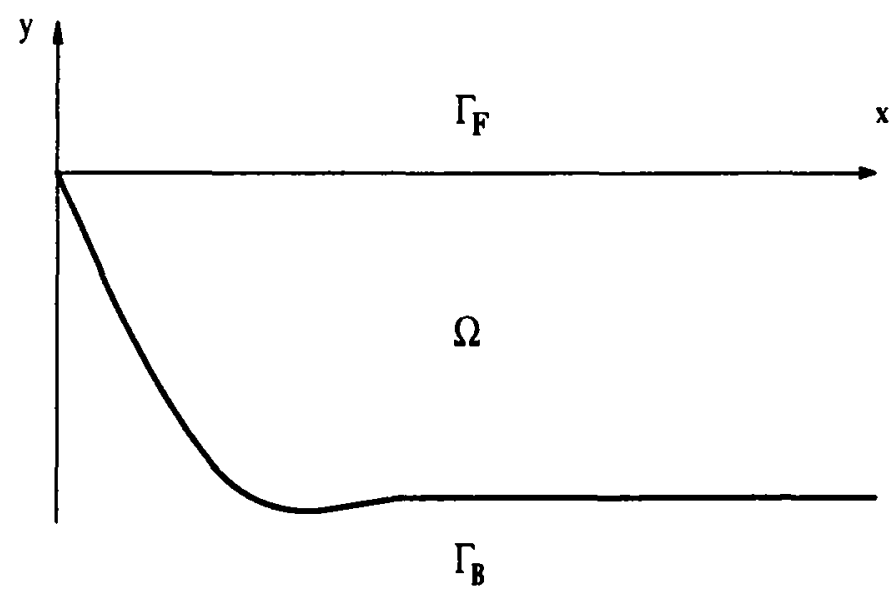

Figure 2.1: The domain of propagation.

As the flow is irrotational, the velocity field $v(x, t)$ in $\Omega$ is the gradient of a potential :

$$
v=-\nabla \phi
$$

$\phi$ is now the unknown of the problem. The linearization of classical fluid dynamics equations ([29]) leads to the following set of equations in $\phi$ :

$$
\left\{\begin{array}{lll}
-\Delta \phi=0 & \text { in } \Omega & (2.2)_{1} \\
\frac{\partial \phi}{\partial n}=h & \text { on } \Gamma_{B} & (2.2)_{2} \\
g \frac{\partial \phi}{\partial n}+\frac{\partial^{2} \phi}{\partial t^{2}}=0 & \text { on } \Gamma_{F} & (2.2)_{3}
\end{array}\right.
$$


Equation (2.2) $)_{1}$ traduces the incompressibility assumption while equation $(2.2)_{3}$ is the free boundary equation and equation $(2.2)_{2}$ expresses the fact that the normal displacement of the bottom $\Gamma_{B}$ is described by a function whose time derivative is equal to $h(x, y, t),(x, y) \in$ $\Gamma_{B}$. In (2.2) $g$ denotes the gravity acceleration; $h(x, y, t)$ appears as the source of the waves.

In order to get a well posed problem we have to prescribe initial conditions for $\phi$ on $\Gamma_{F}$ :

$$
\begin{cases}\phi(x, 0,0)=\phi_{0}(x) & x \in \Gamma_{F} \\ \frac{\partial \phi}{\partial t}(x, 0,0)=\eta_{0}(x) & x \in \Gamma_{F}\end{cases}
$$

Knowing $\phi(x, y, t)$, we can describe the shape of the surface of the ocean, at a given time $t$, by the equation $y=\eta(x, t)$ where the function $\eta(x, t)$ is given by :

$$
\eta(x, t)=\frac{1}{g} \frac{\partial \phi}{\partial t}(x, 0, t)
$$

The mathematical theory of problem $((2.2),(2.3))$ is now well-known. We refer the reader to $[6],[14]$ and $[17]$ for a detailed analysis. The functional framework is naturally given by the energy associated with the problem :

$$
E(\phi, t)=\frac{1}{2} \int_{\Omega}|\nabla \phi|^{2} d x d y+\frac{1}{2 g} \int_{\Gamma_{F}}\left|\frac{\partial \phi}{\partial t}\right|^{2} d x
$$

in which, if we assume that the constant density of the fluid $\rho$ is equal to 1 for instance,

$$
\begin{array}{ll}
\frac{1}{2} \int_{\Omega}|\nabla \phi|^{2} d x d y & \text { is the kinetic energy } \\
\frac{1}{2 g} \int_{\Gamma_{F}}\left|\frac{\partial \phi}{\partial t}\right|^{2} d x d y=\frac{1}{2} \int_{\Gamma_{F}}|\eta|^{2} d x & \text { is the potential energy }
\end{array}
$$

It is easy to derive the following identity :

$$
\frac{d}{d t} E(\phi, t)=\frac{1}{2} \int_{\Gamma_{F}} h \frac{\partial \phi}{\partial t} d \sigma
$$

which shows in particular that, in absence of a source $(h=0)$, the energy is constant in time.We shall make about $\Omega$ the following geometrical assumption :

$\Omega$ is bounded in the $y$-direction.

This assumption expresses that, in some sense, we are interested in the problem with finite depth. The identity (2.5) is the main tool for establishing the following existence and uniqueness theorem. 
Theorem 2.1 If $\Omega$ has the property (2.6) and the data $\left(\phi_{0}, \eta_{0}, h\right)$ of the problem satisfy:

$$
\begin{cases}\cdot & \left(\phi_{0}, \eta_{0}\right) \in H^{1 / 2}\left(\Gamma_{B}\right) \times L^{2}\left(\Gamma_{B}\right) \\ \cdot & h \in W^{1,1}\left(0, T ; H^{-1 / 2}\left(\Gamma_{B}\right)\right)\end{cases}
$$

then problem ((2.2),(2.3)) has a unique finite energy solution $\phi$ which satisfies :

$$
\text { . } \phi \in C^{0}\left(0, T ; H^{1}(\Omega)\right) \cap C^{1}\left(0, T ; L^{2}(\Omega)\right) \text {. }
$$

In its mathematical nature, problem $((2.2),(2.3))$ is very close to second order hyperbolic equations, as the wave equation for instance, but it is not rigorously an hyperbolic problem in the sense that there is no finite propagation velocity property. This is due to the Laplace equation. Nevertheless one can show that if the data $u_{0}, u_{1}$, and $h$ have compact support in space and if $\Omega$ is unbounded in the $x$-direction, then at any time $t>0$, the solution $\phi$ is exponentially decaying in the $x$ direction. This is due to the property of the kernel of the Laplace equation. So in fact, equations $((2.2),(2.3))$ behave "almost" as the ones of a finite propagation phenomenon. The main non standard point is that the propagation phenomenon is driven by the boundary condition (instead of the interior equation for acoustic waves). In the case of a constant depth bottom, the nature of the propagation is easy to describe :

$$
\begin{aligned}
& \Omega=\left\{(x, y) \in l R^{N} \times\right]-L, 0[\} \\
& \Gamma_{F}=\left\{(x, 0), x \in l R^{N}\right\} \\
& \Gamma_{B}=\left\{(x,-L), x \in l R^{N}\right\}
\end{aligned}
$$

With the help of the use of the partial Fourier transform in the $x$-direction :

$$
\hat{\phi}(k, y, t)=\frac{1}{(2 \pi)^{N / 2}} \int \phi(x, y, t) e^{-i k x} d x \quad k \in l R^{N}
$$

it can be shown that any finite energy solution of $((2.2),(2.3))$ can be seen, when $h=0$, as the superposition over wave vectors $k$ of propagating harmonic plane waves in the form :

$$
a(k, y) \exp i(\omega(k) t-k \cdot x)
$$

where the function $\omega=\omega(k)$ satisfies the following dispersion relation :

$$
\omega^{2}=g|k| t h|k| L
$$

which clearly show that the plane waves propagating in a constant depth medium are dispersive : both phase and group velocities do depend on the wave vector $k$. Let us mention that the amplitude function $a(k, y)$ decays exponentially with the depth. 
Constant depth for the water waves equation is the equivalent of constant coefficients in the acoustic wave equation. Therefore variations of the depth are responsible for analogous phenomena to the ones produced by the variations of coefficients in the acoustic wave equation : reflection and transmission of waves, diffraction, ...

For our purpose, the main differences between the acoustic wave equation and the water waves equation can be summarized as follows :

- the propagation velocity is infinite for water waves and finite for acoustic waves

- water waves are dispersive while acoustic waves are not

- in $l R^{N+1}$, the propagation phenomenon only concerns $N$-space dimensions for water waves while it affects the $(N+1)$ space dimensions for acoustic waves.

\section{Absorbing boundary conditions for the problem in two space dimensions}

We shall assume here, without restricting the generality of our study, that the domain $\Omega$ is bounded in the direction $x<0$, unbounded in the direction $x>0$ and that the bottom's depth is constant for large $x$ :

$$
\left\{\begin{array}{l}
\Omega \subset\{(x, y), x>0\} \\
\Omega \cap\{(x, y) / x>R\}=] R,+\infty[\times] 0, L[
\end{array}\right.
$$

which corresponds to the case of figure 2.1 .

We want to bound our domain of calculation in the direction $x>0$ by introducing an artificial frontier at $x=R+\ell$.

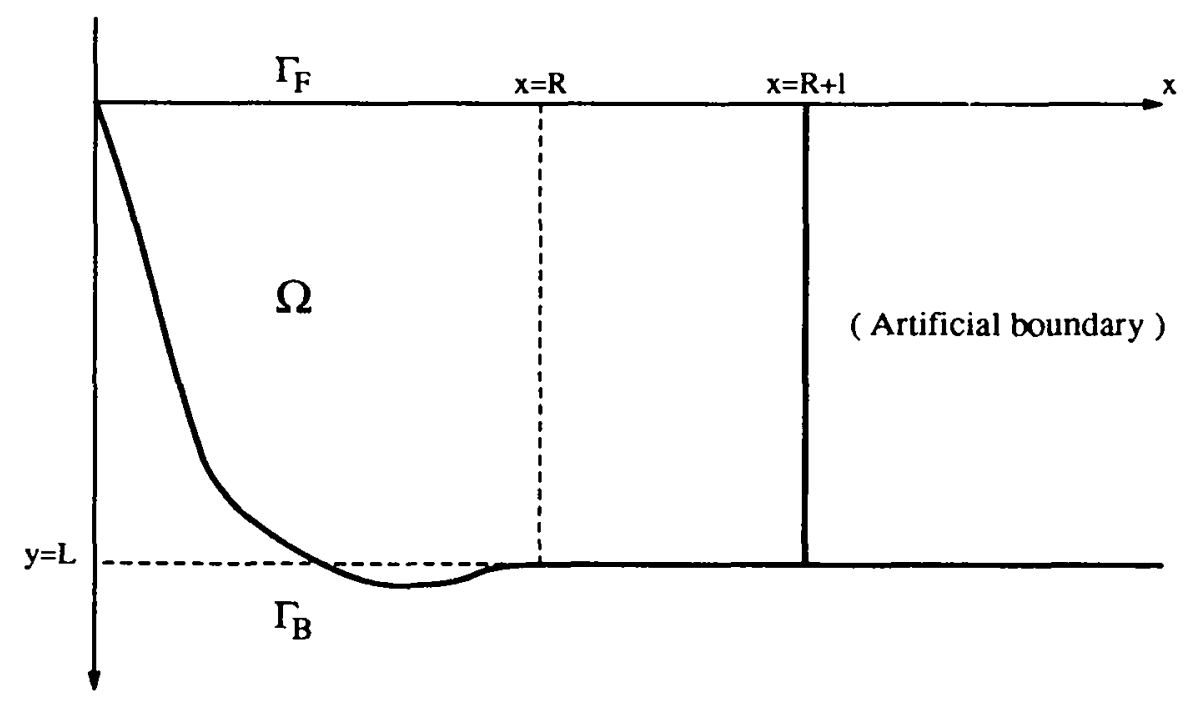


Thus we have to impose an artificial boundary condition at $x=R+\ell$. We shall present the ideal or transparent boundary condition in section 3.1. As such a condition is not easy to treat numerically, we shall construct a family of approximations of this transparent condition, in section 3.2. These will be our absorbing boundary conditions. We analyze the stability of these conditions from a mathematical point of view in section 3.3. In section 3.4, we propose a numerical scheme for the discretization of these conditions and show how they are easy to take into account from a practical point of view.

\subsection{The transparent boundary condition}

The process we are going to follow is very classical (see [13],[15],[19],[20] for other types of equations). We suppose of course that the data of the problem $\left(\phi_{0}, \eta_{0}, h\right)$ have compact support included in the half space $x<R$. In fact, to justify our approach, we shall assume in a first step that :

$$
\left\{\begin{array}{l}
\phi_{0}=\eta_{0}=0 \\
h(x, 0)=\frac{\partial h}{\partial t}(x, 0)=0 \\
\operatorname{supp} h(\cdot, t) \subset\{x<R\}, \text { for } t>0
\end{array}\right.
$$

With these assumptions it is clear that the function $\phi(x, y, t)$ has the following properties:

$$
\left\{\begin{array}{l}
\phi(x, y, 0)=0 \\
\frac{\partial \phi}{\partial t}(x, y, 0)=0
\end{array}\right.
$$

Then, it is easy to see that the problem $((2.2),(2.3))$ is equivalent to a transmission problem. Indeed, let us introduce the following notation :

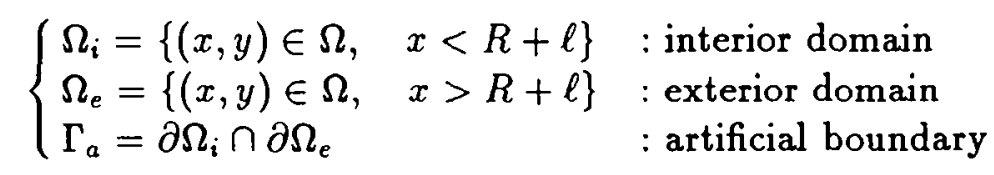

Then $\phi$ is the solution of $((2.2),(2.3))$ if and only if $\left(\phi_{i}, \phi_{e}\right)=\left(\left.\phi\right|_{\Omega_{i}},\left.\phi\right|_{\Omega_{e}}\right)$ is the (unique) solution of the following transmission problem 


$$
\begin{aligned}
& \left\{\begin{array}{l}
\Delta \phi_{i}=0 \quad \text { in } \Omega_{i} \\
\Delta \phi_{e}=0 \quad \text { in } \Omega_{e}
\end{array}\right. \\
& \begin{cases}\frac{\partial^{2} \phi_{i}}{\partial t^{2}}+g \frac{\partial \phi_{i}}{\partial n}=0 \quad \text { on } \Gamma_{F}^{i}=\Gamma_{F} \cap \bar{\Omega}_{i} \\
\frac{\partial^{2} \phi_{e}}{\partial t^{2}}+g \frac{\partial \phi_{e}}{\partial n}=0 \quad \text { on } \Gamma_{F}^{e}=\Gamma_{F} \cap \bar{\Omega}_{e}\end{cases} \\
& \begin{cases}\frac{\partial \phi_{i}}{\partial n}=\mathrm{h} \quad \text { on } \Gamma_{B}^{i}=\Gamma_{B} \cap \bar{\Omega}_{i} \\
\frac{\partial \phi_{e}}{\partial n}=0 \quad \text { on } \Gamma_{B}^{e}=\Gamma_{B} \cap \bar{\Omega}_{e}\end{cases} \\
& \left\{\begin{array}{ll}
\phi_{i}(x, 0,0)=0 \\
\frac{\partial \phi_{i}}{\partial t}(x, 0,0)=0 \\
\phi_{e}(x, 0,0)=0 \\
\frac{\partial \phi_{e}}{\partial t}(x, 0,0)=0
\end{array} \quad x \in \Gamma_{F}^{i}\right.
\end{aligned}
$$

Now let us introduce the operator $B$ acting on functions defined on $\Gamma_{a} \times l R^{+}$(we identify $\Gamma_{a}$ with the segment $\left.[0, L]\right)$

$$
\varphi(y, t) \rightarrow \psi(y, t)=(B \varphi)(y, t)
$$

formally defined by

$$
B \varphi=-\left.\frac{\partial \phi}{\partial x}\right|_{\Gamma_{a}}
$$

where $\phi$ is the unique solution of : 


$$
\begin{cases}\Delta \phi=0 & \text { in } \Omega_{e} \\ \frac{\partial \phi}{\partial n}=0 & \text { on } \Gamma_{B}^{e} \\ \frac{\partial^{2} \phi}{\partial t^{2}}+g \frac{\partial \phi}{\partial n}=0 & \text { on } \Gamma_{F}^{e} \\ \phi(x, 0,0)=0 & \text { on } \Gamma_{F}^{e} \\ \frac{\partial \phi}{\partial t}(x, 0,0)=0 & \text { on } \Gamma_{F}^{e} \\ \left.\phi\right|_{\Gamma_{a}}=\varphi & \text { on } \Gamma_{a}\end{cases}
$$

What we write in this section is formal but could be justified mathematically with the help of an adequate functional framework. This is not our purpose in this paper. Nevertheless let us recall some important properties of $B$, when acting of functions $\varphi(y, t)$ satisfying $\varphi(0, t)=\frac{\partial \varphi}{\partial t}(0, t)=0$ (cf. (3.2)) :

$$
\begin{aligned}
& \text { (i) } B \text { is causal : } \varphi_{1}=\varphi_{2} \text { for } t \leq T \Rightarrow B \varphi_{1}=B \varphi_{2} \text { for } t \leq T \\
& \text { (ii) } \int_{0}^{T} \int_{0}^{L}(B \varphi)(y, t) \frac{\partial \varphi}{\partial t}(y, t) d y d t \geq 0 \quad \forall \varphi, \forall T \geq 0
\end{aligned}
$$

(i) is trivial while (ii) is a consequence of the identity (apply Green's formula):

$$
\begin{aligned}
& \int_{0}^{T} \int_{0}^{L}(B \varphi)(y, t) \frac{\partial \varphi}{\partial t}(y, t) d y d t=E(\phi, T) \\
& E(\phi, T)=\frac{1}{2} \int_{\Omega_{\mathrm{e}}}|\nabla \phi(x, y, T)|^{2} d x d y+\left.\frac{1}{2 g} \int_{\Gamma_{F}^{e}} \frac{\partial \phi}{\partial t}(x, 0, T)\right|^{2} d x
\end{aligned}
$$

where $\phi$ is the solution of (3.4).

By construction, it is clear that the function $\phi_{i}$ is a solution of the following initial boundary value problem :

$$
\begin{cases}\Delta \phi_{i}=0 & \text { in } \Omega_{i} \\ \frac{\partial \phi_{i}}{\partial n}=h & \text { on } \Gamma_{B}^{i} \\ \frac{\partial^{2} \phi_{i}}{\partial t^{2}}+g \frac{\partial \phi_{i}}{\partial n}=0 & \text { on } \Gamma_{F}^{i} \\ \phi_{i}(x, 0,0)=0 & \text { on } \Gamma_{F}^{e} \\ \frac{\partial \phi_{i}}{\partial t}(x, 0,0)=0 & \text { on } \Gamma_{F}^{e} \\ \frac{\partial \phi_{i}}{\partial x}+B \phi_{i}=0 & \text { on } \Gamma_{a}\end{cases}
$$


To state that the boundary condition :

$$
\frac{\partial \phi_{i}}{\partial x}+B \phi_{i}=0 \text { on }\left(\Gamma_{a}\right)
$$

is a transparent condition for our problem it is sufficient to prove that the solution of problem is unique. Indeed, the uniqueness is a direct consequence of the positivity property (3.6) of $B$ since we have the following energy identity (for any $T \geq 0$ ) :

$$
\begin{aligned}
& E\left(\phi_{i}, T\right)+\int_{0}^{T} \int_{\Gamma_{a}} B \phi_{i} \frac{\partial \phi_{i}}{\partial t} d y d t=E(0)+\int_{0}^{T} \int_{\Gamma_{B}^{i}} h \frac{\partial \phi_{i}}{\partial t} d \sigma d t \\
& E\left(\phi_{i}, T\right)=\frac{1}{2} \int_{\Omega_{i}}\left|\nabla \phi_{i}(x, y, T)\right|^{2} d x d y+\frac{1}{2 g} \int_{\Gamma_{F}^{i}}\left|\frac{\partial \phi_{i}}{\partial t}(x, 0, T)\right|^{2} d x
\end{aligned}
$$

Thus, $\phi_{i}$ is entirely characterized by equations (3.7) and (3.8) is a transparent boundary condition.

It is possible, because of the particular geometry of $\Omega_{e}$ (all that we have written up to now is in fact very general) to get a quasi explicit representation of $B$ with the help of :

$$
\left\{\begin{array}{l}
\text { - a Fourier transform in time } \\
\text { - a modal decomposition in the transverse variable } y
\end{array}\right.
$$

Let us introduce the time Fourier transforms of the functions $\varphi(y, t)$ and $\phi(x, y, t)$ appearing in (3.5):

$$
\begin{aligned}
& \hat{\varphi}(y, \omega)=\frac{1}{\sqrt{2 \pi}} \int_{-\infty}^{+\infty} \varphi(x, t) e^{-i \omega t} d t \\
& \hat{\phi}(x, y, \omega)=\frac{1}{\sqrt{2 \pi}} \int_{-\infty}^{+\infty} \phi(x, y, t) e^{-i \omega t} d t
\end{aligned}
$$

Applying Fourier in time to (3.5) shows that :

$$
\forall \omega \in l R \quad(\widehat{B \varphi})(y, \omega)=\hat{B}(\omega) \hat{\varphi}(\cdot, \omega)
$$

where the operator $\hat{B}(\omega)$ acts on functions of the only space variable $y$ ( $\hat{\varphi}$ is defined on $\Gamma$ ):

$$
\hat{B}(\omega) \hat{\varphi}=-\left.\frac{\partial}{\partial x}(\hat{\phi}(\omega))\right|_{\Gamma_{a}}
$$

where $\hat{\phi}(\omega)$ is the outgoing solution of the problem

$$
\begin{cases}\Delta \hat{\phi}(\omega)=0 & \text { in } \Omega_{e} \\ \frac{\partial \hat{\phi}}{\partial n}(\omega)=0 & \text { on } \Gamma_{B}^{e} \\ g \frac{\partial \hat{\phi}}{\partial n}(\omega)+\omega^{2} \hat{\phi}(\omega)=0 & \text { on } \Gamma_{F}^{e} \\ \left.\hat{\phi}(\omega)\right|_{\Gamma_{a}}=\hat{\phi}(\omega) & \text { on } \Gamma_{a}\end{cases}
$$


Problem (3.12) is not elliptic. It is to the water wave equation what Helmholtz equation is to the acoustic wave equation. The outgoing solution of (3.12) can be defined with the help of the limiting absorption principle (see [32],[33]) :

$$
\hat{\phi}(\omega)=\lim _{\varepsilon \searrow 0} \hat{\phi}_{\varepsilon}(\omega) \quad\left(\text { in } L_{l o c}^{2}\left(\Omega_{e}\right)\right)
$$

where $\hat{\phi}(\omega)$ is the unique solution of

$$
\left\{\begin{array}{l}
\Delta \hat{\phi}_{\varepsilon}(\omega)=0 \\
\frac{\partial \hat{\phi}_{\varepsilon}}{\partial n}(\omega)=0 \\
g \frac{\partial \hat{\phi}_{\varepsilon}}{\partial n}(\omega)-\left(\omega^{2}+i \varepsilon\right) \hat{\phi}_{\varepsilon}(\omega)=0 \\
\left.\hat{\phi}_{\varepsilon}(\omega)\right|_{\Gamma_{a}}=\hat{\phi} \\
\hat{\phi}_{\varepsilon}(\Omega) \in L^{2}\left(\Omega_{e}\right)
\end{array}\right.
$$

Note that $\phi_{e}(\omega)$ can be alternatively characterized by radiation conditions (when $x \rightarrow+\infty$ ) that we shall give later.

Now we notice that the unbounded operator $A(\omega)$ defined by :

$$
\begin{aligned}
& D(A(\omega))=\left\{\hat{\varphi} \in H^{2}(0, L) / \frac{\partial \hat{\varphi}}{\partial y}(L)=0, \frac{\partial \dot{\varphi}}{\partial y}(0)=\frac{\omega^{2}}{g} \hat{\varphi}(0)\right\} \\
& A(\omega) \hat{\varphi}=-\frac{d^{2} \hat{\varphi}}{d y^{2}}, \quad \forall \hat{\varphi} \in D(A(\omega))
\end{aligned}
$$

is a selt adjoint operator in $L^{2}\left(\Gamma_{a}\right)$ with compact resolvent. Therefore, there exists an Hilbert space basis of $L^{2}(0, L)$ made of eigenfunctions of $A(\omega)$ (whose spectrum is purely a spectral point). The determination of the eigenvalues and of the eigenfunctions of $A(\omega)$ is a trivial exercise on ordinary differential equations. One easily shows that :

$$
\sigma(A(\omega))=\left\{\mu(\omega), \lambda_{1}(\omega), \lambda_{2}(\omega), \ldots, \lambda_{n}(\omega), \ldots\right\}
$$

where $\mu(\omega)$ is the unique negative eigenvalue of $A(\omega)$ and is defined by

$$
\mu(\omega)=-\xi(\omega)^{2}
$$

where $\xi(\omega)$ is the unique solution (see figure 3.1 ) of the equation :

$$
\xi \operatorname{th} \xi L=\frac{\omega^{2}}{g} \quad \xi>0
$$




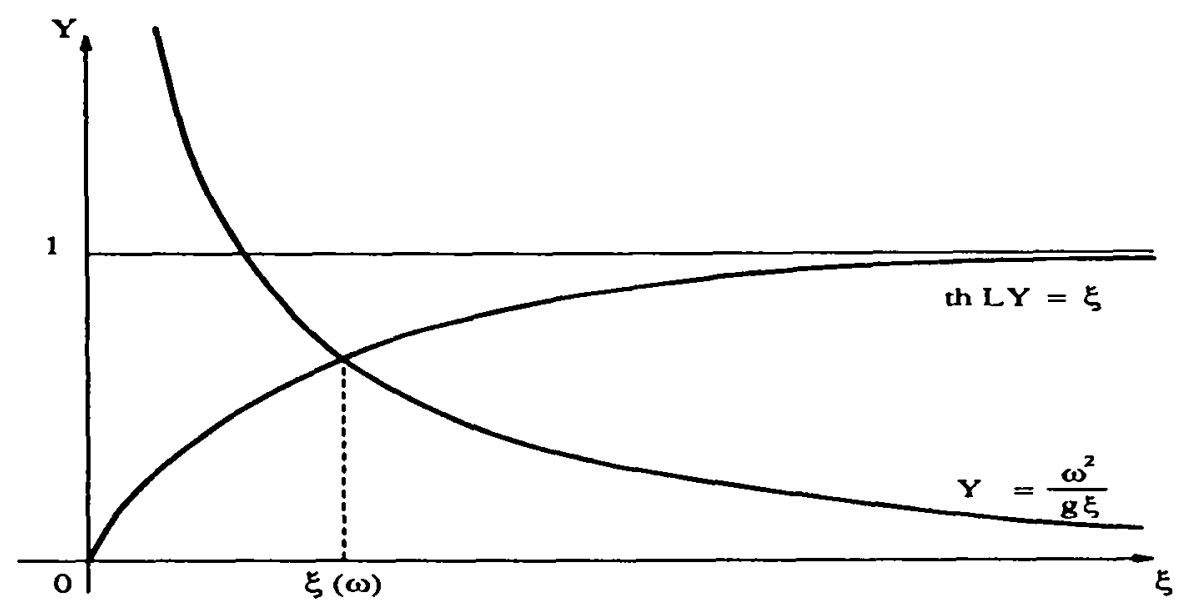

Figure 3.1 : Graphic resolution of (3.14).

$\lambda_{n}(\omega)$ is the $\mathrm{n}^{\text {th }}$ positive eigenvalue of $A(\omega)$ and is defined by

$$
\lambda_{n}(\omega)=k_{n}(\omega)^{2}
$$

where $k_{n}(\omega)$ is the unique solution of :

$$
k \operatorname{tg} k L=-\frac{\omega^{2}}{g} \quad n \pi-\frac{\pi}{2} \leq k L \leq n \pi+\frac{\pi}{2}
$$

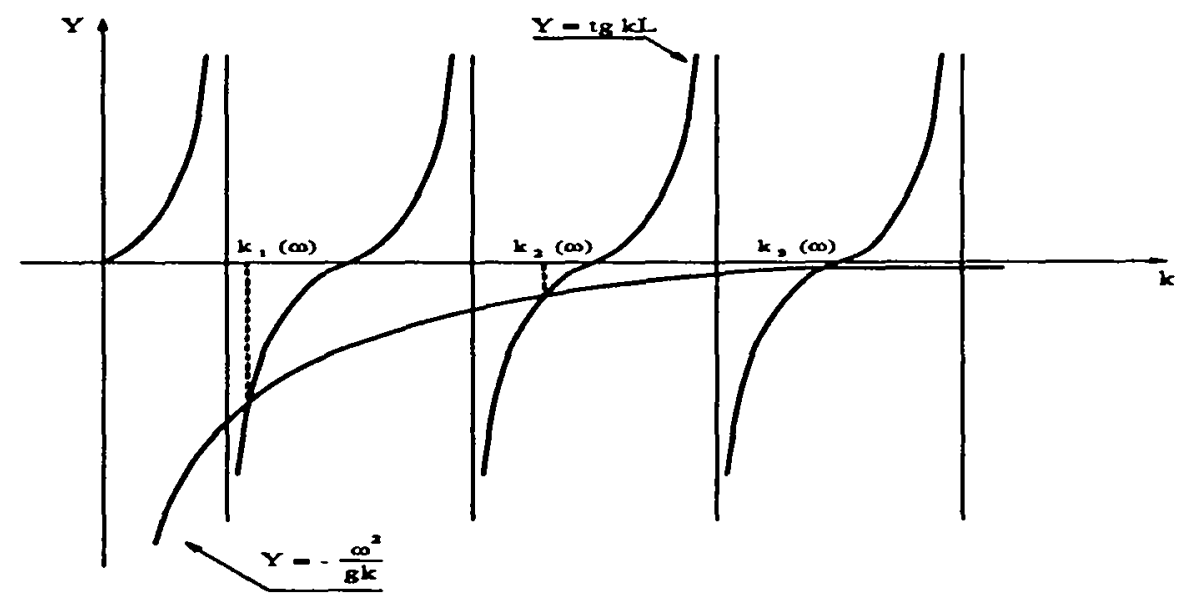

Figure 3.2 : Graphic resolution of (3.16).

Moreover a complete set of orthonormal eigenfunctions of $A(\omega)$ is given by :

$$
\left\{w_{0}(\omega), w_{1}(\omega), \ldots, w_{n}(\omega), \ldots\right\}
$$


where

$$
\left\{\begin{array}{l}
A(\omega) w_{0}(\omega)=\mu(\omega) w_{0}(\omega) \\
A(\omega) w_{n}(\omega)=\lambda_{n}(\omega) w_{n}(\omega)
\end{array}\right.
$$

These functions are given by :

$$
\begin{cases}w_{0}(\omega, y)=a_{0}(\omega) \operatorname{ch}(\xi(\omega) y) & a_{0}(\omega)=\left\{\frac{L}{2}+\frac{\operatorname{sh} 2 \xi(\omega) L}{4 \xi(\omega)}\right\}^{1 / 2} \\ w_{n}(\omega, y)=a_{n}(\omega) \cos \left(k_{n}(\omega) y\right) & a_{n}(\omega)=\left\{\frac{L}{2}+\frac{\sin 2 k_{n}(\omega) L}{4 k_{n}(\omega)}\right\}^{1 / 2}\end{cases}
$$

Now, as $\hat{\psi}$ belongs to $L_{\text {loc }}^{2}\left(\Omega_{e}\right)$, for each $x>R+\ell$, the function $y \rightarrow \hat{\psi}(x, y, \omega)$ belongs to $L^{2}\left(\Gamma_{a}\right)$ and can thus be expanded on the basis $\left\{w_{n}(\omega, \cdot), n \in l N\right\}$

$$
\begin{aligned}
& \hat{\psi}(x, y, \omega)=\sum_{n=0}^{+\infty} \hat{\psi}_{n}(x, \omega) w_{n}(\omega, y) \\
& \int_{0}^{L}|\hat{\psi}(x, y, \omega)|^{2} d y=\sum_{n=0}^{+\infty}\left|\hat{\psi}_{n}(x, \omega)\right|^{2}<+\infty
\end{aligned}
$$

and it is easily seen that each of the functions $\hat{\psi}_{n}(x, \omega)$ satisfies a second order ordinary differential equation in the variable $x$

$$
\begin{gathered}
\left\{\begin{array}{c}
\frac{d^{2} \hat{\psi}_{0}}{d x^{2}}+\xi(\omega)^{2} \hat{\psi}_{0}=0 \quad x>0 \\
\hat{\psi}_{0}(0, \omega)=\hat{\varphi}_{0}(\omega)
\end{array}\right. \\
\left\{\begin{array}{l}
-\frac{d^{2} \hat{\psi}_{n}}{d x}+k_{n}^{2}(\omega) \hat{\psi}_{n}=0 \quad x>0 \quad n \geq 1 \\
\hat{\psi}_{n}(0, \omega)=\hat{\varphi}_{n}(\omega)
\end{array}\right.
\end{gathered}
$$

where we have made the change of variable $x \rightarrow x-(R+\ell)$ and where $\left\{\hat{\varphi}_{n}(\omega), n=\right.$ $0,1,2, \ldots\}$ are the coefficients of $\hat{\varphi}(\omega, \cdot)$ on the basis $\left\{w_{n}(\omega), n=0,1,2, \ldots\right\}$

$$
\hat{\varphi}(\omega, \cdot)=\sum_{n=0}^{+\infty} \hat{\varphi}_{n}(\omega) w_{n}(\omega, \cdot)
$$

With the help of the limiting absorption principle, it is easy to prove that the outgoing solution $\hat{\psi}$ of $(3.4)$ corresponds to the following solution of $((3.18),(3.19))$ :

$$
\left\{\begin{array}{l}
\hat{\phi}_{0}(\omega, x)=\varphi_{0}(\omega) \exp (-i \xi(\omega) x) \\
\hat{\phi}_{n}(\omega, x)=\varphi_{n}(\omega) \exp \left(-k_{n}(\omega) x\right)
\end{array}\right.
$$

which shows that $\hat{\phi}$ can be alternatively characterized by the following radiation condition

$$
\begin{cases}\frac{d \hat{\phi}_{0}}{d x}+i \xi(\omega) \hat{\phi}_{0}=0 & (x \rightarrow+\infty) \\ \hat{\phi}_{n} \rightarrow 0 & (x \rightarrow+\infty)\end{cases}
$$


Reconstructing the function $\phi$ by summation over the index $n$ and inverse Fourier transform in time permits us to write that

$$
\left\{\begin{array}{l}
\phi(x, y, t)=\phi_{0}(x, y, t)+\sum_{n=1}^{+\infty} \phi_{n}(x, y, t) \\
\phi_{0}(x, y, t)=\frac{1}{\sqrt{2 \pi}} \int_{-\infty}^{+\infty} \hat{\varphi}_{0}(\omega) w_{0}(\omega, y) \exp i(\omega t-\xi(\omega) x) d \omega \\
\phi_{n}(x, y, t)=\frac{1}{\sqrt{2 \pi}} \int_{-\infty}^{+\infty} \hat{\varphi}_{n}(\omega) w_{n}(\omega, y) \exp -k_{n}(\omega) x \exp i \omega t d \omega
\end{array}\right.
$$

where $\hat{\varphi}_{0}(\omega)$ and $\hat{\varphi}_{n}(\omega)$ are calculated from $\varphi(y, t)$ by :

$$
\hat{\varphi}_{n}(\omega)=\frac{1}{\sqrt{2 \pi}} \int_{-\infty}^{+\infty} \int_{0}^{L} \varphi(y, t) w_{n}(\omega, y) e^{i \omega t} d y d t .
$$

Formula (3.22) shows that the solution $\phi$ of (3.6) can be interpreted as the superposition of :

- a family of harmonic plane waves propagating in the direction $x>0$ (which explains the name of outgoing solution) $: \phi_{0}(x, y, t)$

- a countable set of families of harmonic evanescent waves $\left\{\phi_{n}(x, y, t)\right\}$ whose penetration distances are $\left\{k_{n}(\omega)^{-1}\right\}$.

From (3.20), we can now give a representation of the operator $B(\omega)$ which is a diagonal operator in the basis $\left\{w_{n}(\omega), n \in l N\right\}$ since :

$$
\left\{\begin{array}{l}
B(\omega) w_{0}(\omega)=i \xi(\omega) w_{0}(\omega) \\
B(\omega) w_{n}(\omega)=k_{n}(\omega) w_{n}(\omega)
\end{array}\right.
$$

Therefore

$$
\hat{\varphi}=\sum \varphi_{n}(\omega) \hat{w}_{n}(\omega) \stackrel{B(\omega)}{\longrightarrow} i \xi(\omega) \hat{\varphi}_{0}(\omega) \hat{w}_{0}(\omega)+\sum_{n=1}^{+\infty} k_{n}(\omega) \hat{\varphi}_{n}(\omega) w_{n}(\omega) .
$$

By inverse Fourier transform in time, we can now give a representation of the operator $B$

$$
\left\{\begin{array}{l}
\varphi(y, t) \stackrel{B}{\longrightarrow} \psi(y, t)=(B \varphi)(y, t)=\psi_{0}(y, t)+\sum_{n=1}^{+\infty} \psi_{n}(y, t) \\
\psi_{0}(y, t)=\frac{1}{\sqrt{2 \pi}} \int_{-\infty}^{+\infty} \int_{0}^{L} i \xi(\omega) w_{0}(y, \omega) w_{0}\left(y^{\prime}, \omega\right) \varphi\left(y^{\prime}, t\right) e^{i \omega t} d y d \omega \\
\psi_{n}(y, t)=\frac{1}{\sqrt{2 \pi}} \int_{-\infty}^{+\infty} \int_{0}^{L} k_{n}(\omega) w_{n}(y, \omega) w_{n}\left(y^{\prime}, \omega\right) \varphi\left(y^{\prime}, t\right) e^{i \omega t} d y^{\prime} d \omega
\end{array}\right.
$$

Formulas (3.23) clearly shows that the operator $B$ is non local with respect to space and time variable. In fact it appears formally as an integral operator 


$$
\begin{aligned}
& (B \varphi)(y, t)=\int_{0}^{t} \int_{0}^{L} K\left(y, y^{\prime} ; t-s\right) \varphi\left(y^{\prime}, s\right) d y^{\prime} d s \\
& K(y, \xi ; t)=K_{0}\left(y, y^{\prime} ; t\right)+\sum_{n=1}^{+\infty} K_{n}\left(y, y^{\prime} ; t\right) \\
& K_{0}\left(y, y^{\prime}, t\right)=\frac{1}{\sqrt{2 \pi}} \int_{-\infty}^{+\infty} i \xi(\omega) w_{0}(y, \omega) w_{0}\left(y^{\prime}, \omega\right) e^{i \omega t} d t \\
& K_{n}\left(y, y^{\prime}, t\right)=\frac{1}{\sqrt{2 \pi}} \int_{-\infty}^{+\infty} k_{n}(\omega) w_{n}(y, \omega) w_{n}\left(y^{\prime}, \omega\right) e^{i \omega t} d t
\end{aligned}
$$

(In fact, it is a singular integral operator and the integrals have to be understood as principal values). Moreover, as for instance the functions $\xi(\omega)$ and $k_{n}(\omega)$ are not rational fractions of $i \omega, B$ cannot be expressed with the help of differential operators. That is why we need, in order to treat efficiently the artificial boundary, to use "simple" (from a numerical point of view) approximations of $B$.

Before constructing these approximations, let us make a parallel with what happens with the $2 \mathrm{D}$ acoustic wave equation when the artificial frontier is a line $x=$ cste.

- the expansion on eigenfunctions $w_{n}(\omega, \cdot)$ is replaced by the use of the partial Fourier transform in the $x$ direction $: \varphi(x) \rightarrow \hat{\varphi}(k)$

- the Fourier variable $k$ (physically the $x$ wave number) is the equivalent of the index $n$

- the transparent boundary condition is written $\frac{\partial u}{\partial x}+B_{\mathrm{o}} u=0$, where $B_{\mathrm{o}}$ is a non local operator which is diagonal in $(k, \omega)$ variables :

$$
\begin{cases}\left(\widehat{B_{\circ} \varphi}\right)(k, \omega)=i \omega\left(1-\frac{k^{2}}{\omega^{2}}\right)^{1 / 2} \hat{\varphi} & \\ \left(1-\frac{k^{2}}{\omega^{2}}\right)^{1 / 2}=\sqrt{1-\frac{k^{2}}{\omega^{2}}} & \text { if }|k| \leq|\omega| \\ \left(1-\frac{k^{2}}{\omega^{2}}\right)^{1 / 2}=-i \sqrt{\frac{k^{2}}{\omega^{2}}-1} & \text { if }|k|>|\omega|\end{cases}
$$

- the region $|k|<|\omega|$ corresponds to the index $n=0$ : propagative plane waves.

- the region $|k|>|\omega|$ corresponds to all the indices $n \geq 1$ : evanescent waves.

\subsection{Construction of the absorbing boundary conditions}

For the acoustic wave equation, the basic idea for the construction of absorbing boundary conditions is the approximation by a rational fraction with real coefficients of the function $i \omega\left(1-\frac{k^{2}}{\omega^{2}}\right)^{1 / 2}$ defined by (3.24). As such an approximation is not possible uniformly in the whole $(k, \omega)$-plane, one ignores the region $|k|>|\omega|$ which corresponds to evanescent waves and tries to get a good approximation for $|k| \leq|\omega|$. Such an approach is completely 
justified as soon as the artificial boundary is not too close to the support of the data of the problem. The equivalent in our case consists in neglecting all the indices $n=1,2, \ldots,+\infty$ which correspond to evanescent waves. This corresponds to assume that, if $\varphi$ is the trace of the solution $\phi$ on $\Gamma_{a}$, then :

$$
\hat{\varphi}(y, \omega) \simeq \hat{\varphi}_{0}(\omega) w_{0}(\omega, y) .
$$

In practice, such an approximation can be made valid by playing with the position of the artificial boundary since one easily sees that, for $n \geq 1$ :

$$
\int_{0}^{L}\left|\hat{\phi}_{n}(R+\ell, y, \omega)\right|^{2} d y \leq e^{-\pi \frac{\ell}{L}} \int_{0}^{L}\left|\hat{\phi}_{n}(R, y, \omega)\right|^{2} d y
$$

uniformly with respect to $n$.

So, under the approximation (3.26), we can write :

$$
B(\omega) \hat{\varphi}(y, \omega) \simeq B_{0}(\omega) \hat{\varphi}(y, \omega)=i \xi(\omega) \hat{\varphi}(y, \omega) .
$$

Now, if $B_{0}$ denotes the operator defined by :

$$
\left(\widehat{B_{0} \varphi}\right)(y, \omega)=B_{0}(\omega) \hat{\varphi}(y, \omega)
$$

an approximate transparent condition is given by :

$$
\frac{\partial \phi}{\partial x}+B_{0} \phi=0 \quad \text { on } \Gamma_{a}
$$

This condition is now local in space but still non local in time because of the dispersive nature of propagative water waves. Moreover, as $\xi(\omega)$ is not a rational fraction with respect to the variable $i \omega, B_{0}$ cannot be expressed with the help of differential operators, which would be very practical from a numerical point of view. The natural idea is now to approximate $\xi(\omega)$ by rational fractions. In order to do this in an adequate way, it is important to describe the main properties of the function $\xi(\omega)$.

For simplicity, we shall assume that the two first time derivatives of functions $\phi$ that we consider on $\Gamma$ are identically 0 :

$$
\phi(y, 0)=\frac{\partial \phi}{\partial t}(y, 0)=0 .
$$

This is natural in the context of absorbing boundary conditions and expresses the fact that $\phi$ is causal and sufficiently regular at $t=0$ (cf. (3.2)). Moreover, under this assumption we know that, by Fourier transform, we have the equalities :

$$
\begin{aligned}
& \left(\frac{\partial \phi}{\partial t}\right)(y, \omega)=i \omega \hat{\phi}(y, \omega) \\
& \left(\frac{\widehat{\partial}^{2} \phi}{\partial t^{2}}\right)(y, \omega)=-\omega^{2} \hat{\phi}(y, \omega)
\end{aligned}
$$




\subsubsection{Properties of the function $\xi(\omega)$}

With the change of variables :

$$
\left\{\begin{array}{l}
(x, y) \rightarrow\left(\frac{x}{L}, \frac{y}{L}\right) \\
t \rightarrow t \sqrt{\frac{g}{L}}
\end{array}\right.
$$

we can assume, without loss of generality that $k=1$, and $g=L=1$.

Therefore the function $\xi(\omega)$ is defined by :

$$
\xi(\omega) \operatorname{th} \xi(\omega)=\omega^{2} \quad \omega \cdot \xi(\omega)>0
$$

from which we easily see that we can write

$$
\xi(\omega)=\omega f(\omega)
$$

where the functions $f(\omega)$ has the following properties :

$$
\begin{cases}\bullet f(\omega)=f(-\omega) & \forall \omega \in l R \\ \bullet \omega \rightarrow f(\omega) & \text { is increasing and } C^{\infty} \text { for } \omega>0 \\ \bullet f(0)=1 & \\ \left.\bullet \lim _{\omega \rightarrow+\infty}\{f(\omega))-|\omega|\right\}=0 & \text { exponentially }\end{cases}
$$

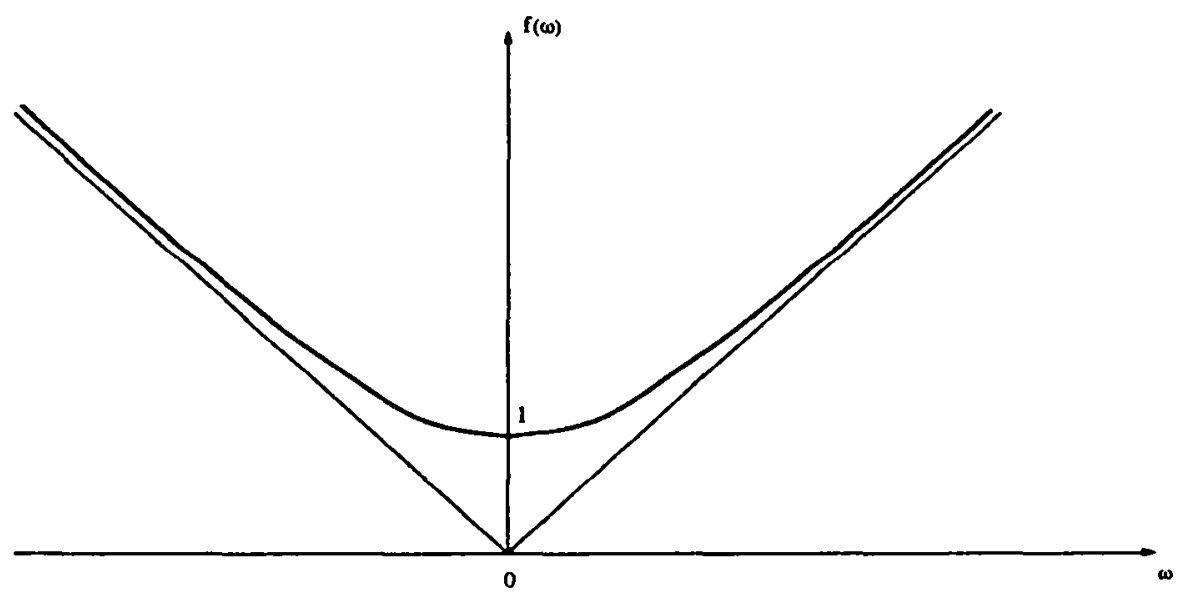

Figure 3.3 : Variations of the function $\omega \rightarrow f(\omega)$.

Note that if $S$ denotes the time-convolution operator whose symbol is $f(\omega)$ then $B_{0}$ is given by :

$$
B_{0}=S \frac{\partial \varphi}{\partial t}
$$




\subsubsection{Rational approximations of the function $f(\omega)$}

Let $R(z)$ be a rational fractional with real coefficients. We want $R(i \omega)$ to be a reasonable approximation (in a sense that we shall precise later) fo $f(\omega)$. As $f(\omega)$ is even, it is natural to impose that $R(i \omega)=Q\left(\omega^{2}\right)$ where $Q$ is also a rational fraction with real coefficients. Moreover we want that the operator whose symbol is $Q\left(\omega^{2}\right)$ be a causal operator. This imposes that the poles of $Q(z)$ are real and positive. All these considerations lead us naturally to consider rational approximations in the form :

$$
f(\omega) \simeq f_{n}(\omega)=1+\sum_{p=1}^{n} \frac{b_{p} \omega^{2}}{1-a_{p} \omega^{2}}
$$

where $\left(a_{p}, b_{p}\right)$ are real coefficients that we want to satisfy, for reasons which shall become clear later, the following conditions :

$$
1-\sum_{p=1}^{n} \frac{b_{p}}{a_{p}}>0 .
$$

A priori, in order that $f_{n}(\omega)$ be the symbol of a causal operator, the coefficients $a_{p}$ should satisfy :

$$
a_{p}>0 \quad \forall p=1,2, \ldots, n \text {. }
$$

We shall see in the sequel that, after having reinterpreted the approximate problem (i.e. the problem with absorbing boundary conditions) in terms of a system of differential equations, this problem still makes sense if we allow to the coefficients $a_{p}$ to be negative. The only difference is that the presence of negative coefficients $a_{p}$ will allow a (controlled) exponential growth of the energy of the solution with the time. Note that :

- $f_{n}=f(0)$ by construction. Therefore one can expect a good approximation of $f(\omega)$ by $f_{n}(\omega)$ for small values of $\omega$ (i.e. for low frequencies).

- Looking at the respective behaviours of $f_{n}(\omega)$ and $f(\omega)$ it is clear that one can't expect to get a uniform approximation of $f(\omega)$ by $f_{n}(\omega)$ on the whole real line, but only in some interval $\left[-\omega^{*}, \omega^{*}\right]$. In particular, in the case where the coefficients $a_{p}$ are positive, the number $\omega^{*}$ is necessarily limited by $\underset{1 \leq p \leq n}{\operatorname{Min}} a_{p}^{-1 / 2}$.

- the condition (3.31) means $\lim _{n \rightarrow+\infty} f_{n}(\omega)>0$. The interest of this condition comes from the stability analysis we shall develop in section 3 .

Of course, we could have considered other approximations of $f(\omega)$. The experience we have on other kinds of problems (see [2],[10],[11]) led us a priori to this choice. We shall discuss a little bit on this point later. Let us now denote by $S_{n}$ the operator defined by :

$$
\left(\widehat{S_{n} \varphi}\right)(\omega)=f_{n}(\omega) \hat{\varphi}(y, \omega)
$$


and introduce the operator $B_{n}$

$$
B_{n} \varphi=S_{n} \frac{\partial \varphi}{\partial t}=\frac{\partial}{\partial t}\left(S_{n} \varphi\right)
$$

which will constitute our approximation of the operator $B$, so that an absorbing boundary condition for problem $((2.2),(2.3))$ can be written

$$
\frac{\partial \phi}{\partial x}+B_{n} \phi=\frac{\partial \phi}{\partial x}+S_{n} \frac{\partial \phi}{\partial t}=0 .
$$

The principal interest of the operator $S_{n}$ stems up from the fact that its evaluation can be reduced to the resolution of a (simple) system of (linear) ordinary differential equations. Indeed, we can write :

$$
S_{n} \varphi=\varphi-\sum b_{p} \psi_{p}
$$

where the auxiliary unknown function $\psi_{p}$ are the solutions of the following differential system :

$$
\left\{\begin{array}{l}
a_{p} \frac{\partial^{2} \psi_{p}}{\partial t^{2}}+\psi_{p}=\frac{\partial^{2} \varphi}{\partial t^{2}} \\
\psi_{p}(y, 0)=\frac{\partial \psi_{p}}{\partial t}(y, 0)=0
\end{array}\right.
$$

To obtain (3.36) and (3.37) it suffices to apply the inverse Fourier transform to (3.33) and to use the properties of the Fourier transform with respect to time derivation. 


\section{The approximate problem}

Using equation (3.35) as an absorbing boundary condition, the problem to be solved in the interior domain $\Omega_{i}$ (with the dimensionless variables $(x, y, t)$ ) is given by :

$$
\begin{cases}\Delta \phi_{i}=0 & \text { in } \Omega_{i} \\ \frac{\partial^{2} \phi_{i}}{\partial t^{2}}+\frac{\partial \phi_{i}}{\partial n}=0 & \text { on } \Gamma_{F}^{i} \\ \frac{\partial \phi_{i}}{\partial n}=h & \text { on } \Gamma_{B}^{i} \\ \frac{\partial \phi_{i}}{\partial x}+S_{n} \frac{\partial \phi_{i}}{\partial t}=0 & \text { on } \Gamma_{a} \\ \phi_{i}(x, 0,0)=0 & \text { on } \Gamma_{F}^{i} \\ \frac{\partial \phi_{i}}{\partial t}(x, 0,0)=0 & \text { on } \Gamma_{F}^{i}\end{cases}
$$

which is equivalent to find $\left(\phi_{i}(x, y, t) ; \psi_{p}(y, t), 1 \leq p \leq n\right)$ such that :

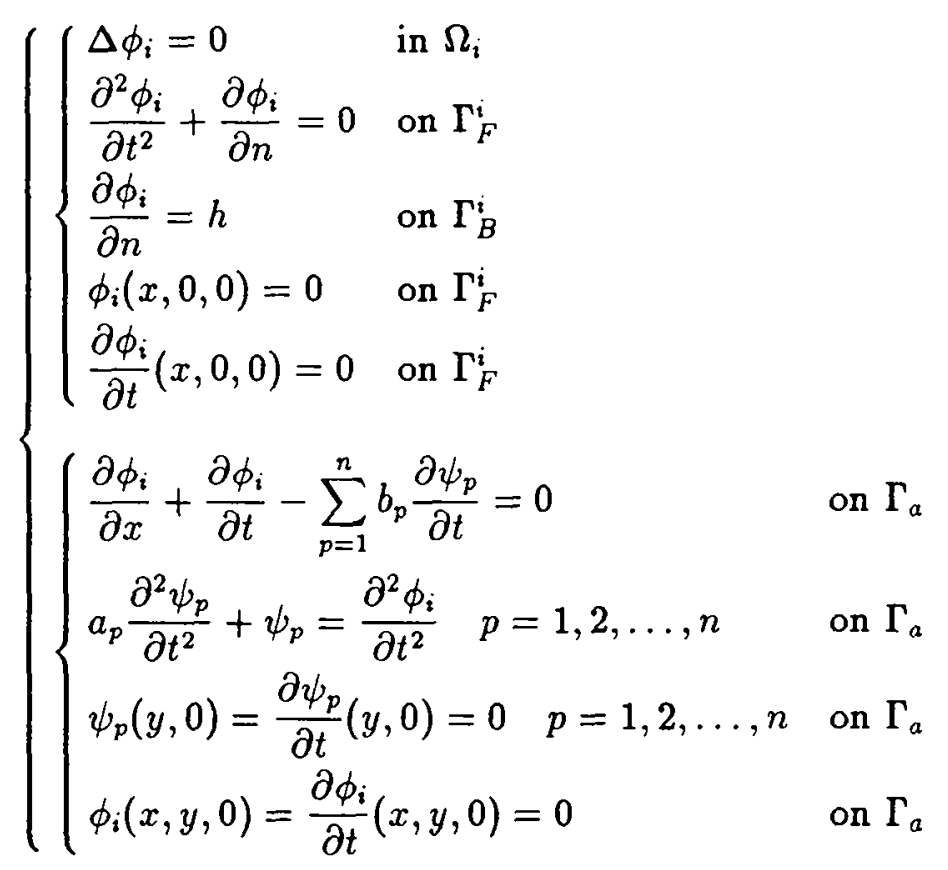

Let us make some comments about this approximate problem :

- When one comes back to the original variables $(x, y, t)$, the equations on the boundary $\Gamma_{a}$ take the following form :

$$
\left\{\begin{array}{l}
\frac{\partial \phi_{i}}{\partial x}+\frac{1}{\sqrt{g} \bar{L}} \frac{\partial \phi_{i}}{\partial t}-\sqrt{\frac{L}{g}} \sum_{p=1}^{n} b_{p} \frac{\partial \psi_{p}}{\partial t}=0 \\
a_{p} \frac{\partial^{2} \psi_{p}}{\partial t^{2}}+\frac{g}{L} \psi_{p}=\frac{\partial^{2} \phi_{i}}{\partial t^{2}}
\end{array}\right.
$$


If $n=0$ (which means that $f(\omega)$ is approximated by $f(0)$ ), the auxiliary functions $\psi_{p}$ disappear and the boundary condition takes the form :

$$
\frac{\partial \phi_{i}}{\partial x}+\frac{1}{\sqrt{g L}} \frac{\partial \phi_{i}}{\partial t}=0
$$

which is exactly the form of a transparent boundary condition for the $1 \mathrm{D}$ wave equation with velocity $c=\sqrt{g L}$. This is not surprising since, from dispersion relation (2.7), we see that $\sqrt{g L}$ is the phase velocity (and also the group velocity) of harmonic water waves at low frequencies.

The presence of the term $\sum b_{p} \frac{\partial \psi_{p}}{\partial t}$ and of the equation $(3.40)_{2}$ expresses the fact that we take into account the dispersive nature of water waves.

- There are several ways of writing initial value problems equivalent to (3.38). It suffices to change the definition of the auxiliary unknown functions $\psi_{p}$. The form (3.39) appeared to us as one of the most natural in view of a numerical discretization (see sect. 3.4)

- In the general case, there is no reason for the data $\left(\varphi_{0}, \eta_{0}, h\right)$ of the problem to satisfy :

$$
\left\{\begin{array}{l}
\varphi_{0}=\eta_{0}=0 \\
h(x, y, 0)=\frac{\partial h}{\partial t}(x, y, 0)=0
\end{array}\right.
$$

In that case we have to work with the new unknown functions :

$$
\tilde{\phi}(x, y, t)=\phi(x, y, t)-\left(\phi_{0}(x, y) a_{0}(t)+\phi_{1}(x, y) a_{1}(t)\right)
$$

where $\phi_{0}$ and $\phi_{1}$ are the solutions of the elliptic problems $\left(h_{0}(x, y)=h(x, y, 0)\right.$, $\left.h_{1}(x, y)=\frac{\partial h}{\partial t}(x, y, 0), \varphi_{1}(x)=g \eta_{0}(x)\right)$

$$
\begin{cases}\Delta \phi_{j}=0 \quad \text { in } \Omega & j=1,2 \\ \left.\phi_{j}\right|_{\Gamma_{F}}=\varphi_{j} & j=1,2 \\ \left.\frac{\partial \phi_{j}}{\partial n}\right|_{\Gamma_{F}}=h_{j} & j=1,2\end{cases}
$$

and $a_{0}(t)$ and $a_{1}(t)$ are regular functions of time such that :

$$
\begin{cases}a_{0}(0)=1 & a_{0}^{\prime}(0)=0 \\ a_{1}(0)=0 & a_{1}^{\prime}(0)=1\end{cases}
$$


Then $\tilde{\phi}$ is solution of the following problem :

$$
\begin{cases}\Delta \tilde{\phi}=0 & \text { in } \Omega \\ \frac{\partial^{2} \tilde{\phi}}{\partial t^{2}}+g \frac{\partial \tilde{\phi}}{\partial n}=0 & \text { on } \Gamma_{F} \\ \frac{\partial \tilde{\phi}}{\partial n}=\tilde{h} & \text { on } \Gamma_{B} \\ \tilde{\phi}(x, 0,0)=0 & \text { on } \Gamma_{F} \\ \frac{\partial \tilde{\phi}}{\partial t}(x, 0,0)=0 & \text { on } \Gamma_{F}\end{cases}
$$

where the function $\tilde{h}$ is given by

$$
\tilde{h}(x, y, t)=\left(h(x, y, t)-a_{0}(t) h_{0}(x, y)-a_{1}(t) h_{1}(x, y)\right) .
$$

We can then apply our absorbing boundary conditions for the approximation of $\dot{\phi}$.

\subsection{Stability analysis of the initial boundary value problem}

The minimal mathematical analysis to be done in this kind of work must be devoted to prove the well-posedness of the approximate problem. This problem in our case, namely problem (3.39), is not so classical, since although it is of hyperbolic nature, the well-known theory developed by Kreiss for hyperbolic systems ([24]) cannot be applied here.

Well posedness of (3.39) would be straightforward if we were able to prove that the approximate operator $B_{n}$ has the same positivity property as $B$. We did not succeed in proving such a property, except in the "case $n=0$ ", which corresponds to the boundary condition

for which we easily get the energy identity :

$$
\frac{\partial \phi_{i}}{\partial t}+\frac{\partial \phi_{i}}{\partial x}=0
$$

$$
\frac{1}{2} \frac{d}{d t}\left(\int_{\Omega_{i}}\left|\nabla \phi_{i}\right|^{2} d x d y+\int_{\Gamma_{F}^{i}}\left|\frac{\partial \phi_{i}}{\partial t}\right|^{2} d x\right)+\int_{\Gamma_{a}}\left|\frac{\partial \phi_{i}}{\partial t}\right|^{2} d y=0 .
$$

One could think also adapt the method of Kreiss, namely a modal analysis, to our specific problem. With such an approach, it would be possible a priori to get necessary and sufficient conditions but there are two major drawbacks :

- it leads to the problem of locating the zeros of a countable set of transcendental equations in the complex plane, which is a priori difficult.

- such an approach is a priori restricted to the case where $\Omega_{i}$ is a rectangle.

We have finally chosen an approach which is intermediate between the modal analysis and the energy techniques.

First of all, to get some insights about the final results it appears useful to us to consider a simplified problem. 


\subsubsection{Stability analysis of a simplified problem}

Let us consider the problem posed in the half space $x<0$ (namely $l R_{-}^{2}$ ):

$$
\begin{cases}\Delta \phi(x, y)=0 & \text { for } x<0 \\ \frac{\partial \phi}{\partial x}+\frac{\partial \phi}{\partial t}-\sum_{p=1}^{n} b_{p} \frac{\partial \psi_{p}}{\partial t}=0 & \text { at } x=0 \\ a_{p} \frac{\partial^{2} \psi_{p}}{\partial t^{2}}+\psi_{p}=\frac{\partial^{2} \phi}{\partial t^{2}} & \text { at } x=0 \\ + \text { initial conditions at } t=0, x=0 & \end{cases}
$$

Such a problem is obtained from the equations of problem (3.38) when one forgets the two physical boundaries (the bottom $\Gamma_{B}^{i}$ and the free boundary $\Gamma_{F}^{i}$ ). It is natural to ask to this problem to be well posed since one could consider that the part of the solution which is located far enough from these two boundaries is not influenced by them and is approximately governed by (3.41).

Lemma 3.1 Problem (3.41) is well-posed in $L^{2}\left(l R_{-}^{2}\right)$ if and only if

$$
1-\sum_{p=1}^{n} \frac{b_{p}}{a_{p}} \geq 0 .
$$

Proof : We assume that the initial data of problem (3.41), namely :

$$
\begin{cases}\phi(0, y, 0)=\varphi_{0}(y) & y \in \mathbb{R} \\ \psi(0, y, 0)=\psi_{p}^{0}(y) & y \in l R \\ \frac{\partial \psi_{p}}{\partial t}(0, y, 0)=\psi_{p}^{1}(y) & y \in l R\end{cases}
$$

are elements of $L^{2}(l R)$. With the help of the partial Fourier transform in the direction $y$ :

$$
\begin{aligned}
& \phi(x, y, t) \rightarrow \hat{\phi}(x, \xi, t) \\
& \psi_{p}(y, t) \rightarrow \hat{\psi}_{p}(\xi, t)
\end{aligned}
$$

we can write a problem posed only on the boundary $x=0$ whose unknown are :

$$
\left\{\begin{array}{l}
\varphi(y, t)=\psi(0, y, t) \\
\psi_{p}(y, t) \quad 1 \leq p \leq n
\end{array}\right.
$$

More precisely, $\left(\hat{\varphi}(\xi, t), \hat{\psi}_{p}(\xi, t)\right)$ are solutions of the following set of ordinary differential equations :

$$
\left\{\begin{array}{l}
\frac{d \hat{\varphi}}{d t}-\sum_{p=1}^{n} \frac{d \hat{\psi}_{p}}{d t}+|\xi| \hat{\varphi}=0 \\
a_{p} \frac{d^{2} \hat{\psi}_{p}}{d t^{2}}+\hat{\psi}_{p}=\frac{d^{2} \hat{\varphi}}{d t^{2}}
\end{array}\right.
$$

The factor $|\xi|$ is due to the fact that we have implicitly solved the Laplace equation by partial Fourier transform. We omit the details. 
Let us look for a basis of solutions of (3.42) in the form :

$$
\left\{\begin{array}{l}
\hat{\varphi}=A_{0} e^{s t} \\
\hat{\psi}_{p}=A_{p} e^{s t}
\end{array}\right.
$$

Elementary calculations show that $s$ must be solution of the following characteristic equation :

$$
s\left(1-\sum_{p=1}^{n} \frac{b_{p} s^{2}}{1+a_{p} s^{2}}\right)=-|\xi|
$$

Therefore, proving the stability in $L^{2}$ of (3.41) is equivalent to prove that, for any branch $s(\xi)$ of solutions of $(3.43), \operatorname{Re}(s(\xi))$ remains bounded, uniformly with respect to $\xi$. As the functions $s(\xi)$ are continuous functions of $\xi$, it is sufficient to study their behaviour for large $\xi$. In this goal let us set:

$$
\left\{\begin{array}{l}
\varepsilon=\frac{1}{|\xi|^{2}} \\
s=|\xi| z
\end{array}\right.
$$

which gives a new equation in the variable $z$ :

$$
z\left(1-\sum_{p=1}^{n} \frac{b_{p} z^{2}}{a_{p} z^{2}+\varepsilon}\right)=-1 .
$$

Rewriting (3.44) in polynomial form and passing to the limit when $\varepsilon$ goes to zero, we obtain :

$$
\left\{\begin{array}{l}
z^{2 n}(1+\alpha z)=0 \\
\alpha=1-\sum_{p=1}^{n} \frac{b_{p}}{a_{p}}
\end{array}\right.
$$

Applying the implicit function theorem to (3.44) shows that equation (3.44) admits, for small $\varepsilon$, a branch of solution $z_{0}(\varepsilon)$ which satisfies, if $\alpha \neq 0$ :

$$
z_{0}(\varepsilon)=-\left(1-\sum_{p=1}^{n} \frac{b_{p}}{a_{p}}\right)^{-1}+\left(\sum_{p=1}^{n} \frac{b_{p}}{a_{p^{2}}}\right) \varepsilon+0\left(\varepsilon^{2}\right) .
$$

This means that the corresponding branch of solutions of (3.43), let $s_{0}(\xi)$, satisfy :

$$
s_{0}(\xi)=-\left(1-\sum_{p=1}^{n} \frac{b_{p}}{a_{p}}\right)^{-1}|\xi|+\left(\sum_{p=1}^{n} \frac{b_{p}}{a_{p^{2}}}\right) \frac{1}{|\xi|}+0\left(\frac{1}{|\xi|^{3}}\right) .
$$

To get what happens to the other branches of solutions we first rewrite (3.43) in the form:

$$
\frac{s}{|\xi|}\left\{\prod_{p=1}^{n}\left(1+a_{p} s^{2}\right)-\sum_{p=1}^{n} b_{p} p^{2}\left(\prod_{j \neq p}\left(1+a_{j} s^{2}\right)\right)\right\}=-\prod_{p=1}^{n}\left(1+a_{p} s^{2}\right) .
$$


Taking the limit of (3.47) when $|\xi|$ goes to infinity gives the equation

$$
\prod_{p=1}^{n}\left(1+a_{p} s^{2}\right)=0
$$

Denoting by $\sqrt{z}$ the square root of $z$ with strictly positive real part when $z \neq 0$, the application of the implicit functions theorem to (3.47) proves that for large $|\xi|,(3.47)$ has $2 n$ branches of solutions, namely $\left\{s_{p}^{+}(\xi), s_{p}^{-}(\xi), p=1,2, \ldots, n\right\}$ satisfying :

$$
s_{p}^{ \pm}(\xi)= \pm \frac{i}{\sqrt{a_{p}}}\left(1+\frac{b_{p}}{a_{p}} \frac{2}{|\xi|}\right)+0\left(\frac{1}{|\xi|^{2}}\right) .
$$

The conclusion is now a direct consequence of the two equalities (3.46) and (3.49). (Note that for the limit case $1-\sum_{p=1}^{n} \frac{b_{p}}{a_{p}}=0,(3.46)$ is no longer valid. However it is not difficult to show that in this case $s_{0}(\xi)$ tends to zero when $\left.|\xi| \rightarrow+\infty\right)$.

- When the coefficients $a_{p}$ are all positive, it is not difficult to prove that, under condition (3.31), equation (3.43) has exactly one unique negative real solution and $2 n$ purely imaginary roots, coupled by conjugate pairs. This implies that the corresponding solution of (3.41) remains bounded uniformly with respect to time (even if $t \rightarrow+\infty$ ).

- As soon as one of the coefficients $a_{p}$ is negative, formula (3.49) proves that there will exist some solutions of (3.41) whose $L^{2}$-norm will be exponentially increasing with respect to time (with a growth in approximatively $\exp \left(\sqrt{-a_{p}} t\right)$ ). This is a kind of "weak" instability.

- It is natural to wonder what would happen if we replace by $A(s)$ by a more general rational fraction, containing in particular a polynomial entire part in the form :

$$
P_{m}(s)=a_{m} s^{2 m}+\text { lower order terms. }
$$

Then, it is easy to prove that the characteristic equation $s A(s)+|\xi|=0$ has solutions whose behaviour when $|\xi| \rightarrow \infty$ is given by :

$$
\begin{aligned}
& s_{k}(\xi) \sim\left(a_{m}\right)^{\frac{1}{2 m+1}} \exp \left(\frac{2 i k \pi}{2 m+1}|\xi|^{\frac{1}{2 m+1}}\right) \quad(|\xi| \rightarrow+\infty) \text { if } a_{m}>0 \\
& s_{k}(\xi) \sim\left(-a_{m}\right)^{\frac{1}{2 m+1}} \exp \left(\frac{i k \pi}{m+1}|\xi|^{\frac{1}{2 m+1}}\right) \quad(|\xi| \rightarrow+\infty) \text { if } a_{m}<0
\end{aligned}
$$

So, in each case, there exists some modes of wave number $\xi$ whose amplitude grows exponentially in time with a factor $e^{\lambda|\xi|^{\frac{1}{2 m+1}}} t$ for some $\lambda>0$. Thus the corresponding problem of type (3.41) is ill-posed. Such a result has to be compared with the one obtained in [30] in the more complicated situation of the wave equation. It corresponds to a result of strong ill-posedness since the $L^{2}$ norm of the solution at a given time $t$ cannot be estimated by any (even high order) Sobolev norm of the initial data (see [21] for different definitions of well-posedness in the case of hyperbolic systems). 


\subsubsection{Stability analysis of the complete problem}

In this section if $\Omega$ is an open set of $l R^{N+1}$ and $\Gamma$ a part of the boundary of $\Omega, H^{s}(\Omega)$ and $H^{s}(\Gamma)$, for $s \in l R$, will denote the usual Sobolev spaces defined respectively on $\Omega$ and $\Gamma$ $([1],[25])$. Moreover :

- $\|\cdot\|$ will represent the norm in $L^{2}(\Omega)$

- $|\cdot| \Gamma$ will represent the norm in $L^{2}(\Gamma)$

- $|\cdot|_{s, \Gamma}$ will represent the norm in $H^{s}(\Gamma)$, for $s \neq 0$

Of course, as we consider complex valued functions, we have :

$$
\begin{aligned}
& (\phi, \psi)=\int_{\Omega} \phi \bar{\psi} d x \\
& (\varphi, \psi)_{\Gamma}=\int_{\Gamma} \varphi \bar{\psi} d \sigma
\end{aligned}
$$

We shall analyze problem (3.39) by applying the Laplace transform in space :

$$
\left\{\begin{array}{l}
\phi(x, y, t) \rightarrow \tilde{\phi}(x, y, s)=\frac{1}{\sqrt{2 \pi}} \int_{0}^{+\infty} \phi(x, y, t) e^{-s t} d t \\
s=\eta+i \omega \quad \eta>0, \omega \in l R
\end{array}\right.
$$

It is easy to see that $\bar{\phi}$ is solution of a problem of the following type :

$$
\begin{cases}-\Delta \tilde{\phi}=f & \text { in } \Omega_{i} \\ \frac{\partial \tilde{\phi}}{\partial n}+s^{2} \tilde{\phi}=\tilde{g} & \text { on } \Gamma_{F}^{i} \\ \frac{\partial \tilde{\phi}}{\partial n}=\tilde{h} & \text { on } \Gamma_{B}^{i} \\ \frac{\partial \tilde{\phi}}{\partial x}+s A(s) \tilde{\phi}=0 & \text { on } \Gamma_{a}\end{cases}
$$

where

$$
A(s)=1-\sum_{p=1}^{n} \frac{b_{p} s^{2}}{1+a_{p} s^{2}} .
$$

The difficulty consists in getting estimations on :

$$
\begin{cases}\|\tilde{\phi}(\eta+i \omega)\| & \forall \omega \in l R \\ \|\nabla \tilde{\phi}(\eta+i \omega)\| & \forall \omega \in l R\end{cases}
$$

for a given $\eta>0$. Multiplying first equation of (3.50) by $\bar{s} \overline{\bar{\phi}}$ and integrating over $\Omega_{i}$ gives:

$$
\bar{s}\|\nabla \tilde{\phi}\|^{2}-\left(\frac{\partial \tilde{\phi}}{\partial n}, s \tilde{\phi}\right)_{\partial \Omega}=(\tilde{f}, s \tilde{\phi}) .
$$


Using the boundary conditions, we get :

$$
\begin{aligned}
\bar{s}\|\nabla \tilde{\phi}\|^{2} & +s|s|^{2}|\tilde{\phi}|_{\Gamma_{F}^{i}}^{2}+|s|^{2} A(s)|\tilde{\phi}|_{\Gamma_{a}}^{2} \\
& =(\tilde{f}, s \tilde{\phi})+(\tilde{g}, s \tilde{\phi})_{\Gamma_{F}^{i}}+(\tilde{h}, s \tilde{\phi})_{\Gamma_{B}^{i}} .
\end{aligned}
$$

Taking the real part of (3.51), we get :

$$
\begin{aligned}
\eta\|\nabla \tilde{\phi}\|^{2} & +\eta\left(\eta^{2}+\omega^{2}\right)|\tilde{\phi}|_{\Gamma_{F}^{i}}^{2}+\left(\eta^{2}+\omega^{2}\right) \operatorname{Re}(A(\eta+i \omega))|\tilde{\phi}|_{\Gamma_{a}}^{2} \\
& =\operatorname{Re}\left\{(\tilde{f}, s \tilde{\phi})+(\tilde{g}, s \tilde{\phi})_{\Gamma_{F}^{i}}+(\tilde{h}, s \tilde{\phi})_{\Gamma_{B}^{i}}\right\}
\end{aligned}
$$

So we can get uniform estimates with respect to $\omega$ of $\|\nabla \bar{\phi}(\eta+i \omega)\|$ and $\|\bar{\phi}(\eta+i \omega)\|$ (by Poincaré's inequality) as soon as :

$$
\operatorname{Re}(A(\eta+i \omega)) \geq \alpha>0 \quad \forall \omega \in l R
$$

for some $\alpha>0$ and some $\eta>0$.

Lemma 3.2 The two following propositions are equivalent:

(i) $1-\sum_{p=1}^{n} b_{p} / a_{p}>0$

(ii) $\exists \eta>0, \exists \alpha>0 / \forall \omega \in l R R e(A(\eta+i \omega)) \geq \alpha$

Proof :

$$
\left\{\begin{array}{l}
\operatorname{Re}(A(\eta+i \omega))=1-\sum_{p=1}^{n} b_{p} g_{p}(\eta, \omega) \\
g_{p}(\eta, \omega)=\frac{\eta^{2}-\omega^{2}+a_{p}\left(\eta^{2}+\omega^{2}\right)^{2}}{\left[1+a_{p}\left(\eta^{2}-\omega^{2}\right)\right]^{2}+4 a_{p}^{2} \eta^{2} \omega^{2}}
\end{array}\right.
$$

Let us note that :

$$
\lim _{\omega \rightarrow \infty} g_{p}(\eta, \omega)=\frac{1}{a_{p}}
$$

Therefore

$$
\lim _{\omega \rightarrow \infty} \operatorname{Re}(A(\eta+i \omega))=1-\sum_{p=1}^{n} \frac{b_{p}}{a_{p}}
$$

which shows that (ii) implies (i). 
Now, let us set $c_{p}=a_{p} \eta^{2}$. We have :

$$
\left\{\begin{array}{l}
g_{p}(\eta, \omega)=\frac{1}{a_{p}} G\left(c_{p}, a_{p}\left(\eta^{2}+\omega^{2}\right)\right) \\
G(c, X)=\frac{X^{2}-X+2 c}{X^{2}-2 X+1+4 c}
\end{array}\right.
$$

We have to distinguish two cases :

(i) $c>0$.

As $G(c, X)=1+\frac{1}{X}+0\left(\frac{1}{X}\right)(X \rightarrow \pm \infty)$, it is clear that $G(x, X)$ is decreasing at infinity. Moreover :

$$
\left\{\begin{aligned}
\frac{\partial G}{\partial X}(c, X)=0 & \Leftrightarrow X^{2}-2(1+2 c) X+1=0 \\
& \Leftrightarrow\left\{\begin{array}{l}
X=X_{1}(c)=2 c+1-\sqrt{4 c(1+c)} \\
X=X_{2}(c)=2 c+1+\sqrt{4 c(1+c)}
\end{array}\right.
\end{aligned}\right.
$$

We thus have the following situation :

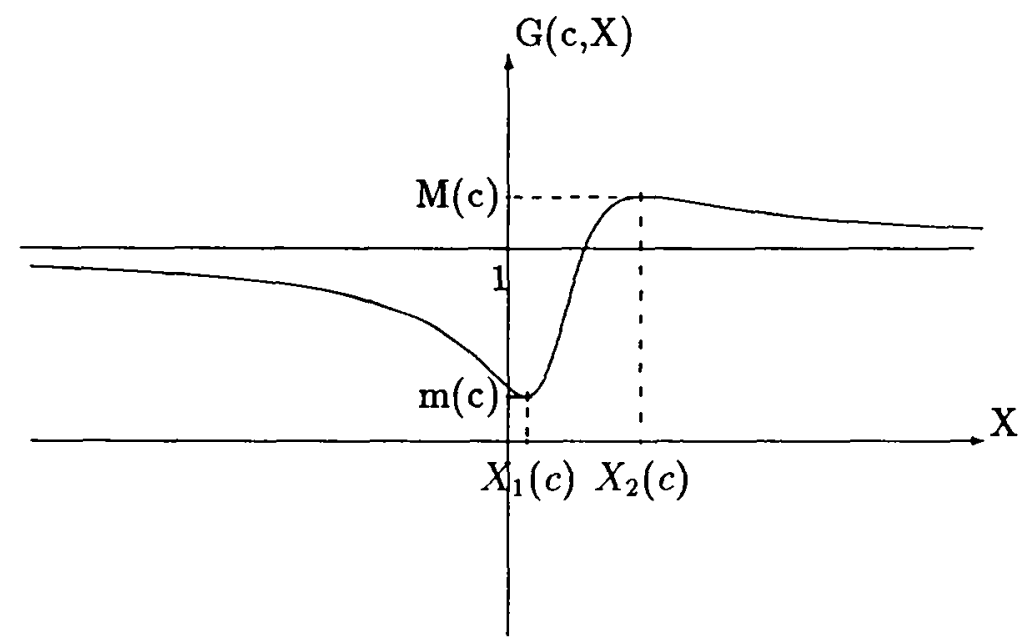

Figure 3.4: Graph of $x \rightarrow G(c, x)(c>0)$

For large $c, X_{1}(c)$ and $X_{2}(c)$ satisfy :

$$
\left\{\begin{array}{l}
X_{1}(c) \sim \frac{1}{4 c} \\
X_{2}(c) \sim 4 c
\end{array} \quad(c \rightarrow+\infty) .\right.
$$


Then, for $c$ large enough we know that :

$$
X_{1}(c)<c<X_{2}(c)
$$

So, as $G(c, c)=\frac{c}{1+c}<1$, that

$$
\forall X \in\left[c,+\infty\left[\frac{c}{1+c} \leq G(c, X) \leq M(c)\right.\right.
$$

where $M(c)$ satisfies :

$$
M(c)=1+\frac{1}{4 c}+0\left(\frac{1}{c^{2}}\right) \quad c \rightarrow+\infty
$$

Applying this result we know that, if $a_{p}>0$ :

$$
\forall \omega \in l R \quad \frac{\eta^{2}}{1+a_{p} \eta^{2}} \leq g_{p}(\eta, \omega) \leq M\left(a_{p} \eta^{2}\right) \cdot \frac{1}{a_{p}}
$$

(ii) $c<-1$

In this case, $G(c, X)$ goes at infinity for two values of $X$ :

$$
X=1 \pm 2 \sqrt{-c}
$$

It is easy to see that one is in the following situation:

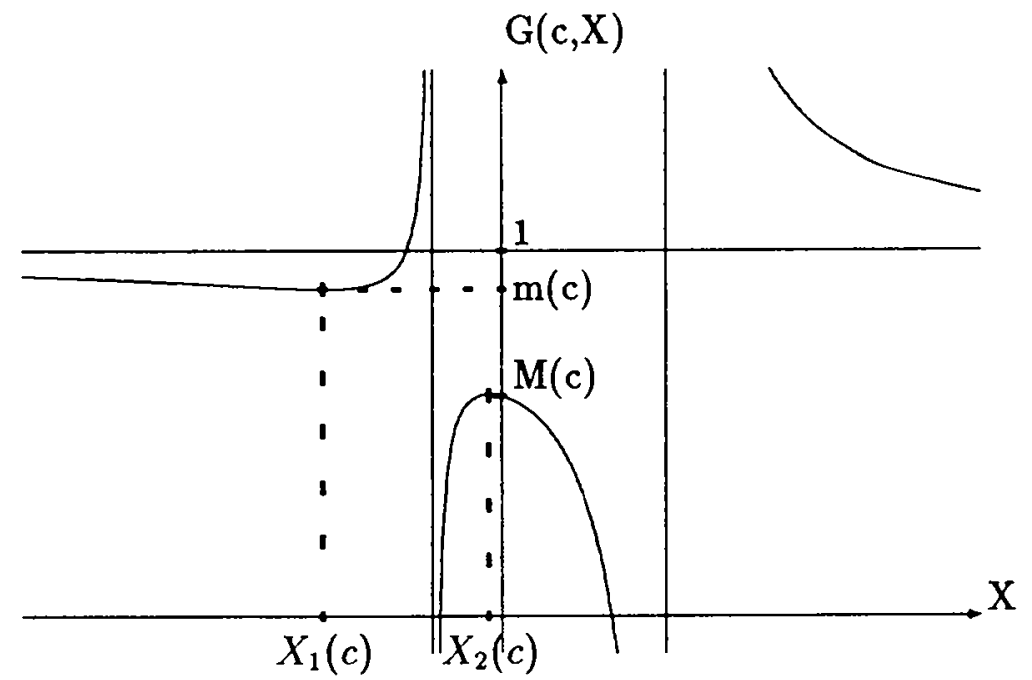

Figure 3.5 : Graph of $x \rightarrow G(c, x)(c<-1)$ 
For large $c, X_{1}(c)$ and $X_{2}(c)$ satisfy :

$$
\left\{\begin{array}{l}
X_{1}(c) \sim 4 c \\
X_{2}(c) \sim \frac{1}{4 c}
\end{array} \quad(c \rightarrow-\infty)\right.
$$

Moreover one checks that $1+2 c<c<1-2 \sqrt{-c}$ so that one deduces that :

$$
\forall X \in\left[-\infty, c\left[\quad m(c) \leq G(c, X) \leq \frac{c}{1+c}\right.\right.
$$

where $m(c)$ satisfies :

$$
m(c)=1+\frac{1}{4 c}+0\left(\frac{1}{c^{2}}\right)
$$

Applying this result we know that for $a_{p}<0$ and $\eta^{2}\left|a_{p}\right|>1$ we get :

$$
\forall \omega \in l R \quad \frac{\eta^{2}}{1+a_{p} \eta^{2}} \leq g_{p}(\eta, \omega) \leq \frac{1}{a_{p}} m\left(a_{p} \eta^{2}\right)
$$

Regrouping now (3.53), (3.56) and (3.58) we can construct two functions $m(\eta)$ and $M(\eta)$ such that, thanks to (3.55) and (3.57) :

$$
\left\{\begin{array}{l}
\lim _{\eta \rightarrow+\infty} m(\eta)=\lim _{\eta \rightarrow+\infty} M(\eta)=1 \\
m(\eta)<M(\eta)
\end{array}\right.
$$

and such that, for $\eta$ large enough,

$$
m(\eta)\left(1-\sum_{p=1}^{n} \frac{b_{p}}{a_{p}}\right)<\operatorname{Re} A(\eta+i \omega)<M(\eta)\left(1-\sum_{p=1}^{n} \frac{b_{p}}{a_{p}}\right) \quad \forall \omega \in l R .
$$

Then, if (i) is satisfied, fixing $\eta$ large enough in order that $m(\eta)>\frac{1}{2}$ (for instance) we get

$$
\operatorname{Re}(A(\eta+i \omega))>\alpha>0
$$

We are now able to prove our main result. 


\section{Theorem 3.1}

(i) If $\left(1-\sum_{p=1}^{n} \frac{b_{p}}{a_{p}}\right)>0$, the initial boundary value problem (3.39) is well posed and its solution $\phi$ satisfies the following estimates (for some $\eta>0$, and any $T>0$ ) :

$$
\begin{aligned}
& \int_{0}^{T} \quad e^{-\eta t}\left\{\|\nabla \phi(t)\|^{2}+\left|\frac{\partial \phi}{\partial t}\right|_{\Gamma_{F}^{i}}^{2}+\left|\frac{\partial \phi}{\partial t}\right|_{\Gamma_{a}}^{2}\right\} d t \\
& \quad \leq C(\eta) \int_{0}^{T} e^{-\eta t}\left\{|\tilde{h}(t)|_{-\frac{1}{2}, \Gamma_{B}^{i}}^{2}+\left|\frac{\partial \tilde{h}}{\partial t}(t)\right|_{-\frac{1}{2}, \Gamma_{B}^{i}}^{2}\right\} d t+C(\eta, T)\left\{\left|\varphi_{0}\right|_{\frac{1}{2}, \Gamma_{F}^{i}}^{2}+\left|\varphi_{1}\right|_{\frac{1}{2}, \Gamma_{B}^{i}}^{2}\right\} .
\end{aligned}
$$

for $\left(\varphi_{0}, \eta_{0}\right)$ given in $H^{1 / 2}\left(\Gamma_{F}^{i}\right)^{2}$ and $h$ in $L_{l o c}^{2}\left(l R^{+} ; H^{-1 / 2}\left(\Gamma_{B}^{i}\right)\right)$.

(ii) If $\left(1-\sum_{p=1}^{n} \frac{b_{p}}{a_{p}}\right)<0$, there exists some cases in which problem (3.39) is ill-posed in the $L^{2}$-sense.

\section{Proof :}

To prove the part (ii) of the theorem, let us consider the following example :

$$
\Omega_{i}=\{(x, y) / x<0,0 \leq y \leq 1\}
$$

and consider particular solutions of (3.39) in the form :

$$
\begin{cases}\phi(x, y, t)=A_{0} \cos \{k(1-y)\} e^{\xi x} e^{s t} & k \in l R \quad \xi>0 \\ \psi_{p}(y, t)=A_{p} \cos \{k(1-y)\} e^{s t} & s \in \mathbb{C}\end{cases}
$$

$\phi$ is harmonic in $\Omega$ as soon as $k=\xi$. The Neuman boundary condition on $\Gamma_{B}^{i}$ is satisfied by construction. It remains to traduce the fact that both boundary conditions on $\Gamma_{F}^{i}$ and $\Gamma_{a}$ must be satisfied.

- the free boundary condition on $\Gamma_{F}^{i}$ is equivalent to :

$$
k \operatorname{tg} k=s^{2}
$$

- the absorbing boundary condition on $\Gamma_{a}$ is equivalent to :

$$
k+s A(s)=0 \text {. }
$$

The set of solutions of equation (3.57) in the quarter plane $\left\{(k, s) \in l R^{+} \times l R^{+}\right\}$can be described as the reunion of a countable set of curves with equation

$$
k=k_{n}(s) \quad n=0,1,2, \ldots
$$

where $k_{n}(s)$ is the unique solution of (3.57) satisfying

$$
n \pi \leq k_{n}(s) \leq n \pi+\frac{\pi}{2}
$$


Moreover, the function $k_{n}(s)$ has the following properties :

$$
\left\{\begin{array}{l}
\bullet s \rightarrow k_{n}(s) \\
\bullet k_{n}(0)=n \pi \\
\bullet \lim _{s \rightarrow+\infty} k_{n}(s)=n \pi+\frac{\pi}{2}
\end{array} \quad\right. \text { is continuous and increasing }
$$

On the other hand the curve $k=-s A(s)$ has an asymptot whose equation is :

$$
k=-\left(1-\sum_{p=1}^{n} \frac{b_{p}}{a_{p}}\right) s .
$$

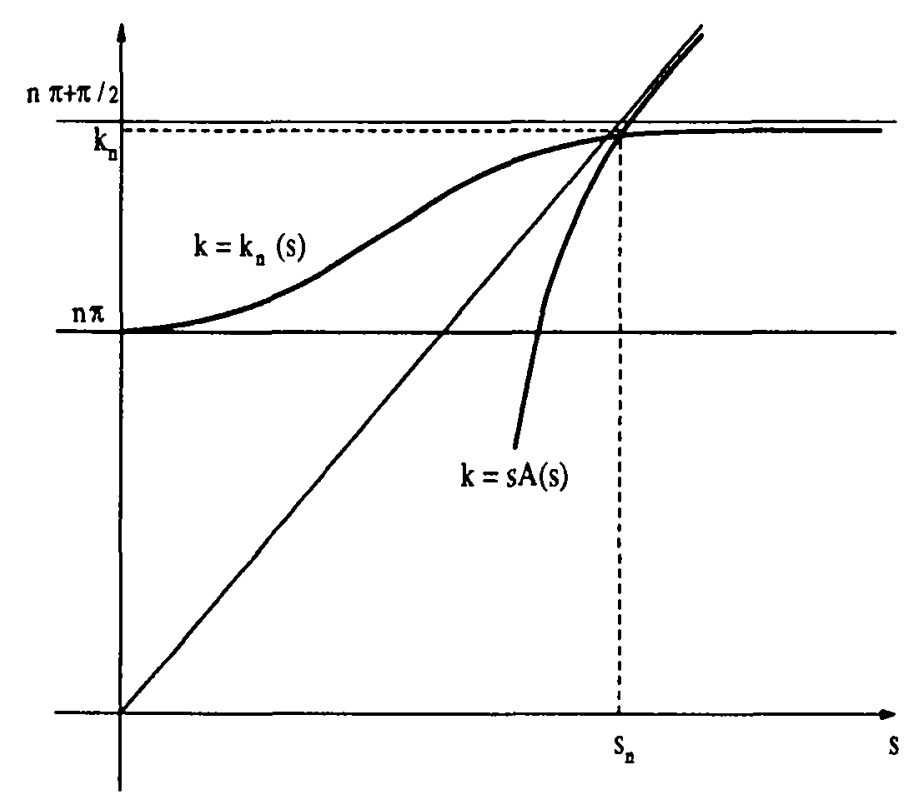

Figure 3.6 : Construction of $\left(s_{n}, k_{n}\right)$.

Therefore it is clear that there exists $n_{0} \in l N$, such that for $n \geq n_{0}$, the system $((3.57),(3.58))$ as a unique solution $\left(s_{n}, k_{n}\right)$ in the strip $\left\{(k, s), n \pi \leq k \leq n \pi+\frac{\pi}{2}\right\}$. Of course both sequences $s_{n}$ and $k_{n}$ go to $+\infty$ with $n$ (even linearly).

Consider now the particular solution of (3.39) given by :

$$
\phi_{n}(x, y, t)=A_{n} \cos k_{n}(1-y) e^{k_{n} x} e^{s_{n} t}
$$

where $A_{n}$, given by :

$$
A_{n}=\frac{1}{2 k_{n}}
$$


has been computed in order that

$$
\left\{\begin{array}{l}
\left\|\phi_{n}(0)\right\|_{L^{2}\left(\Omega_{i}\right)}=1 \\
\left\|\frac{\partial \phi_{n}}{\partial t}(0)\right\|_{L^{2}\left(\Omega_{i}\right)}=s_{n}
\end{array}\right.
$$

We get, for any time $t$

$$
\left\|\phi_{n}(t)\right\|_{L^{2}\left(\Omega_{1}\right)}=e^{s_{n} t}
$$

which proves that problem (3.39) is not well-posed in the $L^{2}$-sense. Note that we have proven that (3.39) is strongly ill-posed since the $L^{2}$-norm of $\phi_{n}(t)$ cannot be estimated by any Sobolev-norm of the initial data, because of the exponential growth.

The proof of part (i) of the theorem is rather standard. First we make the following change of unknown function (without changing the notation)

$$
\phi \rightarrow \phi-\phi_{0} \theta_{0}(t)-\phi_{1} \theta_{1}(t)
$$

where $\phi_{0}$ and $\phi_{1}$ are defined in $H^{1}\left(\Omega_{i}\right)$ by :

$$
\begin{cases}\Delta \phi_{j}=0 & \text { in } \Omega_{i} \\ \left.\phi_{j}\right|_{\Gamma_{F}^{i}}=\varphi_{j} \in H^{1 / 2}\left(\Gamma_{F}^{i}\right) & \\ \left.\phi_{j}\right|_{\Gamma_{a}}=0 & \\ \left.\frac{\partial \phi_{j}}{\partial n}\right|_{\Gamma_{B}^{i}}=0 & \text { on } \Gamma_{F}^{e}\end{cases}
$$

and where $\theta_{0}(t)$ and $\theta_{1}(t)$ are regular functions of time satisfying :

$$
\begin{cases}\theta_{0}(0)=1 & \theta_{0}^{\prime}(0)=0 \\ \theta_{1}(0)=0 & \theta_{1}^{\prime}(1)=1 \\ \operatorname{supp}\left(\theta_{0}, \theta_{1}\right) \subset[0, T] & \end{cases}
$$

We also truncate the Neuman data $h(x, y, t)$ on the interval $[0, T]$ and denote by $\bar{h}(s)$ the Laplace transform of the corresponding truncated function. It is now clear that $\bar{\phi}(s)$ (Laplace transform of the new unknown function $\phi$ ) is solution of problem (3.51) with $\tilde{f}=0$ and $\tilde{g}(s)=\tilde{\theta}_{0}(s)\left(s^{2} \varphi_{0}+\psi_{0}\right)+\tilde{\theta}_{1}(s)\left(s^{2} \eta_{0}+\psi_{1}\right)$, where $\psi_{0}=\left.\frac{\partial \phi_{0}}{\partial n}\right|_{\Gamma_{F}^{i}}$ and $\psi_{1}=\left.\frac{\partial \phi_{1}}{\partial n}\right|_{\Gamma_{F}^{\prime}}$.

We can apply Lemma 3.1 and estimate (3.52) to get :

$$
\begin{aligned}
\eta\|\nabla \tilde{\phi}\|^{2} & +\eta\left(\eta^{2}+\omega^{2}\right)|\tilde{\phi}|_{\Gamma_{F}^{i}}^{2}+\alpha\left(\eta^{2}+\omega^{2}\right)|\tilde{\phi}|_{\Gamma_{a}}^{2} \\
& \leq\left(\eta^{2}+\omega^{2}\right)^{\frac{1}{2}}\left\{|\tilde{g}|_{-\frac{1}{2}, \Gamma_{F}^{i}}|\tilde{\phi}|_{\frac{1}{2}, \Gamma_{F}^{i}}+|\tilde{h}|_{-\frac{1}{2}, \Gamma_{B}^{i}}|\tilde{\phi}|_{\frac{1}{2}, \Gamma_{B}^{i}}\right\}
\end{aligned}
$$

Using the trace theorem in $H^{1}\left(\Omega_{i}\right)$ and Poincarés inequality $([27])$, we know that there exists a constant $C>0$ such that :

$$
\left\{\begin{array}{l}
|\tilde{\phi}|_{\frac{1}{2}, \Gamma_{B}^{i}} \leq C\left(\|\nabla \tilde{\phi}\|+|\tilde{\phi}|_{\Gamma_{F}^{i}}\right) \\
|\tilde{\phi}|_{\frac{1}{2}, \Gamma_{F}^{i}} \leq C\left(\|\left.\nabla \tilde{\phi}|+| \tilde{\phi}\right|_{\Gamma_{F}^{i}}\right)
\end{array}\right.
$$


By Young's inequality, we can find two positive constants $C_{1}(\eta)$ and $C_{2}(\eta)$ such that :

$$
\frac{\eta}{2}\|\nabla \tilde{\phi}\|^{2}+\frac{\eta}{2}\left(\eta^{2}+\omega^{2}\right)|\tilde{\phi}|_{\Gamma_{F}^{i}}^{2}+\alpha\left(\eta^{2}+\omega^{2}\right)|\tilde{\phi}|_{\Gamma_{a}}^{2} \leq\left[C_{1}(\eta)+\omega^{2} C_{2}(\eta)\right]\left(\left.|\tilde{g}|_{-\frac{1}{2}, \Gamma_{F}^{i}}^{2}|+| \tilde{h}\right|_{-\frac{1}{2}, \Gamma_{B}^{2}} ^{2}\right)
$$

Let us set, for $T>0$ :

$$
\begin{array}{lll}
\|h\|_{H^{1}\left(0, T ; H^{-\frac{1}{2}}\left(\Gamma_{B}^{i}\right)\right)}^{2} & =\int_{0}^{T}\left\{|h(t)|_{-\frac{1}{2}, \Gamma_{B}^{i}}^{2}+\left|\frac{\partial h}{\partial t}\right|_{-\frac{1}{2}, \Gamma_{B}^{i}}^{2}\right\} d t \\
\|g\|_{H^{1}\left(0, T ; H^{-\frac{1}{2}}\left(\Gamma_{F}^{i}\right)\right)}^{2} & =\int_{0}^{T}\left\{|g(t)|_{-\frac{1}{2}, \Gamma_{F}^{i}}^{2}+\left|\frac{\partial g}{\partial t}\right|_{-\frac{1}{2}, \Gamma_{F}^{i}}^{2}\right\} d t
\end{array}
$$

By Plancherel's theorem and with causality and truncation arguments $([21],[24])$, we easily get the a priori estimates :

$$
\begin{aligned}
& \int_{0}^{T} e^{-\eta t}\|\nabla \phi(t)\|^{2} d t \leq C(\eta)\left(\|h\|_{H^{1}\left(0, T ; H^{-\frac{1}{2}}\left(\Gamma_{B}^{i}\right)\right)}^{2}+\|g\|_{H^{1}\left(0, T ; H^{\left.-\frac{1}{2}\left(\Gamma_{F}^{i}\right)\right)}\right.}^{2}\right)
\end{aligned}
$$

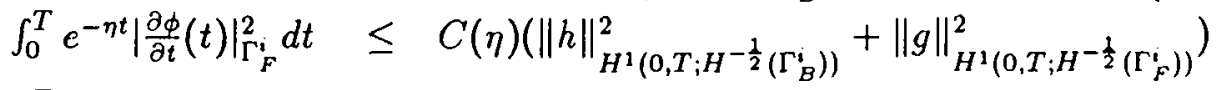

$$
\begin{aligned}
& \int_{0}^{T} e^{-\eta t}\left|\frac{\partial \phi}{\partial t}(t)\right|_{\Gamma_{\mathrm{a}}}^{2} d t \leq C(\eta)\left(\|h\|_{H^{1}\left(0, T ; H^{-\frac{1}{2}}\left(\Gamma_{B}^{i}\right)\right)}^{2}+\|g\|_{H^{1}\left(0, T ; H^{-\frac{1}{2}}\left(\Gamma_{F}^{i}\right)\right)}^{2}\right)
\end{aligned}
$$

To get the final result it suffices to remark that there exists a constant $C(T)$ such that :

$$
\|g\|_{H^{1}\left(0, T ; H^{-\frac{1}{2}}\left(\Gamma_{F}^{i}\right)\right)}^{2} \leq C(T)\left\{\left|\varphi_{0}\right|_{\frac{1}{2}, \Gamma_{i}}^{2}+\left|\eta_{0}\right|_{\frac{1}{2}, \Gamma_{i}}^{2}\right\}
$$

where the constant $C(T)$ can be estimated by the norms of the functions $\theta_{0}(t)$ and $\theta_{1}(t)$ in $H^{3}(0, T)$.

\section{Comments}

- The ill-posedness of (3.39) to more general situations that the particular one we considered here is probably a general result when $1-\sum_{p=1}^{n} \frac{b_{p}}{a_{p}}<0$. The case $1-\sum_{p=1}^{n} \frac{b_{p}}{a_{p}}=0$ is not so clear and would demand a little more analysis...

- Our main estimate is probably not optimal and could certainly be improved, essentially in what concerns the regularity of the initial data. In particular the result should be extended to $\varphi_{0}$ in $L^{2}\left(\Gamma_{F}^{i}\right)$. In this sense, our result could be considered as a weak stability result (compare with the existence theorem 2.1 of section 2 ).

- It is natural to wonder what would happen if we had approximated $f(\omega)$ by a rational fraction including a polynomial part in the form :

$$
P(s)=a_{m} s^{2 m}+\text { lower order terms }
$$

with $a_{m} \neq 0$ and $m \geq 1$. 
In that case, it is not difficult to prove that the model problem in the slab $0 \leq y \leq L$ is strongly ill-posed. Indeed, with the notation of the proof of part (ii) of theorem 3.1, if we look for a particular solution in the form (3.59), $k$ and $s$ much satisfy equations (3.60) and (3.61) with now :

$$
A(s)=a_{m} s^{2 m}+O\left(s^{2 m-2}\right) .
$$

By the implicit function theorem we know that equation (3.60) has a branch of solutions $k_{n}(s)$ for complex $s$ satisfying :

$$
k_{n}(s)=n \pi+\frac{\pi}{2}+O\left(\frac{1}{s^{2}}\right) \quad(|s| \rightarrow+\infty)
$$

then one is reduced to consider for each $n$ the equation :

$$
a_{m} s^{2 m+1}+O\left(s^{2 m-1}\right)=\left(n+\frac{1}{2}\right) \pi+O\left(\frac{1}{s^{2}}\right) .
$$

Still using the implicit function theorem, we know that for $n$ large enough, equation (3.60) has $(2 m+1)$ solutions $s_{n}(\ell), 1 \leq \ell \leq 2 m+1$ such that

$$
\begin{cases}s_{n}(\ell)=\left(a_{m}\right)^{\frac{1}{2 m+1}}\left[\left(n+\frac{1}{2}\right) \pi\right]^{\frac{1}{2 m+1}} e^{i \frac{2 \ell \pi}{2 m+1}}\left(1+\varepsilon_{n}(\ell)\right) & \text { if } a_{m}>0 \\ s_{n}(\ell)=\left(-a_{m}\right)^{\frac{1}{2 m+1}}\left[\left(n+\frac{1}{2}\right) \pi\right]^{\frac{1}{2 m+1}} e^{i \frac{2 \ell \pi}{2 m+1}}\left(1+\varepsilon_{n}(\ell)\right) & \text { if } a_{m}<0\end{cases}
$$

where $\varepsilon_{n}(\ell) \rightarrow 0$ when $n \rightarrow+\infty$. As $m \geq 1$, we can select $\ell$ such that $\cos \frac{2 \ell \pi}{2 m+1}>0$ when $a_{m}<0$ and $\cos \frac{(2 \ell+1) \pi}{2 m+1}<0$ when $a_{m}<0$ and then construct a sequence $\left(s_{n}, k_{n}\right)=$ $\left(s_{n}(\ell), k_{n}\left(s_{n}(\ell)\right)\right)$ of solutions of $(3.60),(3.61)$ such that :

$$
\left\{\begin{array}{l}
\operatorname{Re}\left(s_{n}(\ell)\right)>C \cdot\left[\left(n+\frac{1}{2}\right) \pi\right]^{\frac{1}{2 m+1}}, \quad C>0 \\
k_{n}=n \pi+\frac{\pi}{2}+0
\end{array}\right.
$$

The corresponding solution of the problem in the form (3.59) proves the strong ill posedness.

\subsection{Numerical approximation of the approximate problem}

Our goal in this section is to show how the numerical approximation of problem (3.39) is easy and natural. In particular we shall see that the introduction of the auxiliary functions $\psi_{p}$ due to the absorbing boundary condition does not increase too much the computational cost by comparison with the case of more classical boundary conditions as Neuman or Dirichlet.

The numerical scheme we are going to present is an adaptation of a rather classical implicit scheme which has been analyzed in details in [9] for instance. 
Let us first present the time discretization. We shall use a constant time step $\Delta t$ and denote by $\left(\phi^{n}, \psi_{p}^{n}\right)$ the approximate solution at time $t^{n}=n \Delta t$. The numerical scheme we propose is the following $(0 \leq \theta \leq 1)$ :

$$
\begin{cases}\Delta\left(\theta \phi^{n+1}+(1-2 \theta) \phi^{n}+\theta \phi^{n-1}\right)=0 & \text { in } \Omega_{i} \\ \frac{\phi^{n+1}-2 \phi^{n}+\phi^{n-1}}{\Delta t^{2}}+\theta \frac{\partial \phi^{n+1}}{\partial n}+(1-2 \theta) \frac{\partial \phi^{n}}{\partial n}+\theta \frac{\partial \phi^{n-1}}{\partial n}=0 & \text { on } \Gamma_{F}^{i} \\ \frac{\phi^{n+1}-\phi^{n-1}}{2 \Delta t}-\sum_{p=1}^{N} b_{p} \frac{\psi_{p}^{n+1}-\psi_{p}^{n-1}}{2 \Delta t}+\theta \frac{\partial \phi^{n+1}}{\partial x}+(1-2 \theta) \frac{\partial \phi^{n}}{\partial x}+\theta \frac{\partial \phi^{n-1}}{\partial x}=0 & \text { on } \Gamma_{a} \\ a_{p} \frac{\psi_{p}^{n+1}-2 \psi_{p}^{n}+\psi_{p}^{n-1}}{\Delta t^{2}}+\theta \psi_{p}^{n+1}+(1-2 \theta) \psi_{p}^{n}+\theta \psi_{p}^{n-1}=\frac{\phi^{n+1}-2 \phi^{n}+\phi^{n-1}}{\Delta t^{2}} & \text { on } \Gamma_{a} \\ \theta \frac{\partial \phi^{n+1}}{\partial n}+(1-2 \theta) \frac{\partial \phi^{n}}{\partial n}+\theta \frac{\partial \phi^{n-1}}{\partial n}=\theta h^{n+1}+(1-2 \theta) h^{n}+\theta h^{n-1} & \text { on } \Gamma_{B}^{i}\end{cases}
$$

It is clearly second order accurate in time. (3.65) permits to compute $\phi^{n+1}$ and $\left\{\psi_{p}^{n+1}, 1 \leq\right.$ $p \leq N\}$, knowing $\phi^{n}, \phi^{n-1},\left\{\left(\psi_{p}^{n}, \psi_{p}^{n-1}\right), 1 \leq p \leq N\right\}$. Of course, scheme (3.65) has to be coupled to a start up procedure giving $\phi^{0}$ and $\phi^{1}$. In practice, as $\Delta \phi^{0}=\Delta \phi^{1}=0$, we deduce form the first equation of (3.65) that $\Delta \phi^{n}=0$ at each time step so in fact we solve a Laplace equation at each time step with particular boundary conditions. For the same reasons $\left.\frac{\partial \phi^{n}}{\partial n}\right|_{\Gamma_{B}^{i}}=h^{n}$ for any $n$. What is particularly important from a practical point of view is that the auxiliary unknown $\psi_{p}$ at time $t^{n+1}$ can be eliminated. Indeed, (4.1) can be rewritten in the form :

$$
\left\{\begin{array}{l}
-\Delta \phi^{n+1}=0 \\
\phi^{n+1}+\theta \Delta t^{2} \frac{\partial \phi^{n+1}}{\partial n}=f^{n} \\
\phi^{n+1}+2 \theta \Delta t \frac{\partial \phi^{n+1}}{\partial x}-\sum_{p=1}^{N} b_{p} \psi_{p}^{n+1}=g^{n} \\
a_{p} \psi_{p}^{n+1}+\theta \Delta t^{2} \psi_{p}^{n+1}-\phi^{n+1}=g_{p}^{n} \\
\frac{\partial \phi^{n+1}}{\partial n}=h^{n+1}
\end{array}\right.
$$

where we have set :

$$
\left\{\begin{array}{l}
f^{n}=2 \phi^{n}-\phi^{n-1}-\Delta t^{2}\left[(1-2 \theta) \frac{\partial \phi^{n}}{\partial n}+\theta \frac{\partial \phi^{n-1}}{\partial n}\right] \\
g^{n}=\phi^{n-1}-\sum_{p=1}^{N} b_{p} \psi_{p}^{n-1}-2 \Delta t\left((1-2 \theta) \frac{\partial \phi^{n}}{\partial x}+\theta \frac{\partial \phi^{n-1}}{\partial x}\right) \\
g_{p}^{n}=2 a_{p} \psi_{p}^{n}-a_{p} \psi_{p}^{n-1}-\Delta t^{2}\left[(1-2 \theta) \psi_{p}^{n}+\theta \psi_{p}^{n-1}\right]
\end{array}\right.
$$


Thanks to the local character of the equation relating $\psi_{p}^{n+1}$ and $\phi^{n+1}$ we can eliminate $\psi_{p}^{n+1}$. Indeed we have :

$$
\psi_{p}^{n+1}=\frac{1}{a_{p}+\theta \Delta t^{2}}\left(\phi^{n+1}+g_{p}^{n}\right)
$$

Plugging (3.68) in the second equation of (3.66) shows that $\phi^{n+1}$ is the solution of :

$$
\left\{\begin{array}{l}
-\Delta \phi^{n+1}=0 \\
\frac{\partial \phi^{n+1}}{\partial n}+\frac{1}{\theta \Delta t^{2}} \phi^{n+1}=f^{n} \frac{1}{\theta \Delta t^{2}} \\
\frac{\partial \phi^{n+1}}{\partial n}+\frac{1}{2 \theta \Delta t}\left(1-\sum_{p=1}^{n} \frac{b_{p}}{a_{p}+\theta \Delta t^{2}}\right) \phi^{n+1}=\left(g^{n}+\sum_{p=1}^{N} \frac{b_{p} g_{p}^{n}}{a_{p}+\theta \Delta t^{2}}\right) \frac{1}{2 \theta \Delta t} \\
\frac{\partial \phi^{n+1}}{\partial n}=h^{n+1}
\end{array}\right.
$$

This is a very classical type of problem with Fourier type boundary condition :

$$
\begin{cases}\Delta \phi=0 & \text { in } \Omega_{i} \\ \frac{\partial \phi}{\partial n}+a(x) \phi=q & \text { on } \partial \Omega_{i}\end{cases}
$$

where

$$
a(x)= \begin{cases}1 / \theta \Delta t^{2} & \text { on } \Gamma_{F}^{i} \\ \left(1-\Sigma b_{p} /\left(a_{p}+\theta \Delta t^{2}\right)\right) / 2 \theta \Delta t & \text { on } \Gamma_{a} \\ 0 & \text { on } \Gamma_{B}^{i}\end{cases}
$$

The algorithm is now clear :

(i) Evaluate $g^{n}, g_{p}^{n}, f^{n}$ from $\left(\phi^{n-1}, \phi^{n}, \psi_{p}^{n-1}, \psi_{p}^{n}\right)$ by $(3.67)$

(ii) Solve problem (3.69) to compute $\phi^{n+1}$

(iii) Reactualize the functions $\psi_{p}^{n+1}$ with the help of (3.68).

This algorithm requires some remarks and comments :

- From the stability condition $1-\Sigma \frac{b_{p}}{a_{p}}>0$, we deduce that, at least for $\theta \Delta t^{2}$ small enough :

$$
1-\sum \frac{b_{p}}{a_{p}+\theta \Delta t^{2}}>0
$$

which means that problem (3.69) is elliptic and has a unique solution in $H^{1}\left(\Omega_{i}\right)$.

- In practice, the computation of the auxiliary function $\psi_{p}$ is explicit (see equations (3.68)).

In comparison with the resolution of problem (3.69), its corresponding computational cost is marginal. 
- A priori, the algorithm (phase (i)) implies the numerical evaluation of normal derivatives at the boundary, which is known to be possibly unaccurate if this is not done very carefully. In fact, this can be avoided by using a variational formulation in space and applying a standard finite element method ( $\mathrm{P} 1$ or $\mathrm{Q} 1$ finite elements for instance). That is the method we have applied in practice for the obtention of the numerical results we shall present in section 4 .

- When one uses a classical P1 or Q1 finite element method for the spacial discretization, one gets a numerical scheme which is second order accurate in both space and time. The stability study of such a scheme in the absence of absorbing boundary condition can be found in [9]. The main results can be summarized as follows :

- when $\theta \geq \frac{1}{4}$ the scheme is unconditionally stable

- when $0 \leq \theta<\frac{1}{4}$, the scheme is stable if and only if :

$$
g \frac{\Delta t^{2}}{h} \leq \frac{C}{(1-4 \theta)}
$$

where $C$ is a positive adimensional constant.

In [9], one can also find a dispersion analysis of these schemes, which is a precious guide for the choice of the discretization parameters in order to get the best possible accuracy on the results.

\subsection{Possible strategies for the construction of approximate boun- dary conditions.}

It is natural to consider that we are interested by data $\left(\varphi_{O}, \varphi_{1}\right.$ and $\left.h\right)$ whose frequency content is limited to a band $\left[0, \omega^{*}\right]$. This encourages the fact to privilege the approximation of the function $f(\omega)$ at low frequencies, i.e. in a neighbourhood of $\omega=0$. Particular functions for obtaining such properties are of course the Padé approximations of the function $f(\omega)$. Let us recall that the $(m, m)$ Pade approximant at the origin of the function $f(\omega)$ is the unique rational fraction $\frac{P_{m}(\omega)}{Q_{m}(\omega)}$ whose Taylor expansion as the origin coincides with the one of $f(\omega)$ up to order $\omega^{2 m}$. As an example, for $m=1$, this approximant has the form :

$$
f_{1}(\omega)=1+\frac{b_{1} \omega^{2}}{1-a_{1} \omega^{2}}
$$

Its Taylor development at the origin is given by :

$$
f_{1}(\omega)=1+b_{1} \omega^{2}+a_{1} b_{1} \omega^{4}+0\left(\omega^{6}\right)
$$


Let us recall that the function $f(\omega)$ is defined by :

$$
\left\{\begin{array}{l}
\xi(\omega)=\omega f(\omega) \\
\xi(\omega) \text { th }(\xi(\omega))=\left(\omega^{2}\right)
\end{array}\right.
$$

One finds that its Taylor expansion at the origin is :

$$
f(\omega)=1+\frac{\omega^{2}}{6}+\frac{11}{360} \omega^{4}+0\left(\omega^{6}\right)
$$

By identification between (3.72) and (3.73) we compute that $a_{1}=\frac{11}{60}$ and $b_{1}=\frac{1}{6}$. So:

$$
f_{1}(\omega)=1+\frac{\omega^{2}}{6\left(1-\frac{11 \omega 2}{60}\right)}
$$

Note that :

$$
1-\frac{b_{1}}{a_{1}}=1-\frac{10}{11}=\frac{1}{11}>0
$$

which means that the use of $f_{1}(\omega)$ as an approximation of $f(\omega)$ gives rise to a well posed initial boundary value problem.

A natural strategy would consist to look at the sequence of the diagonal Padé approximants of $f(\omega)$, to examine if they can be written under the general form (3.30) and to see if they lead to well posed problems. As $f(\omega)$ is only known implicitly through a non linear equation, this is a non easy job which demands a lot of simple, but long and tedious calculations. We shall delay this work to a forthcoming paper.

Another method consists in considering the approximation procedure as a minimization problem : for a given number $N$ of simple rational fractions, find the coefficients $a_{k}$ and $b_{k}$ in order to minimize a certain norm of the error function $\left(f_{n}-f\right)(\omega)$ in a given range of frequencies : $\omega \in\left[0, \omega^{*}\right]$. Of course this appears as a constrained optimization problem in $R^{2 N}$ since we have to satisfy the inequality $1-\sum \frac{b_{p}}{a_{p}}<0$. Of course as any optimization algorithm demands an initial guess for the iteration process, one could choose for example the corresponding Padé approximation, which would give in principle a very good approximation of $f(\omega)$ for small values of $\omega$ while the optimization process should make this approximation more uniform in the segment $\left[0, \omega^{*}\right]$ (see $[11]$, for another application of a similar principle).

\section{Numerical results - Validation of the absorbing boun- dary condition}

To validate our method, we consider the following simple model problem :

$$
\Omega=[0, a] \times[0, L]
$$




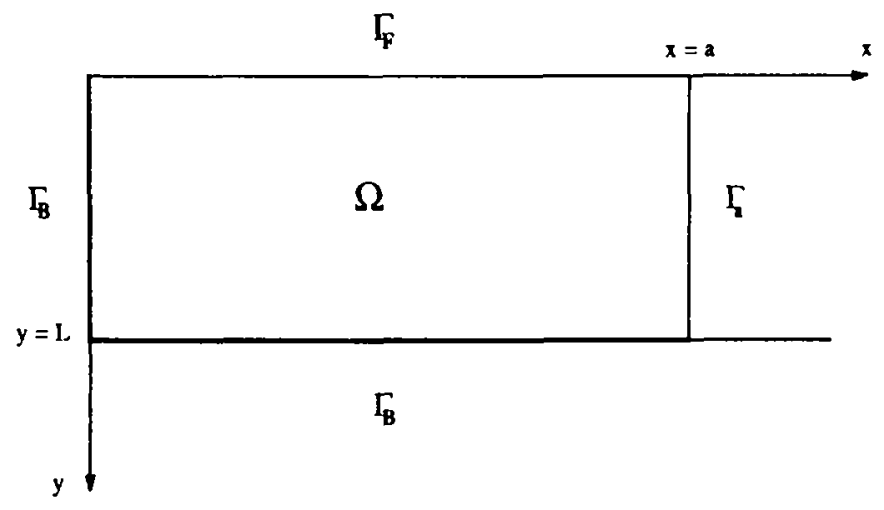

$$
\begin{cases}\Delta \phi=0 & \text { in } \Omega \\ \frac{\partial^{2} \phi}{\partial t^{2}}+g \frac{\partial \phi}{\partial n}=0 & \text { on } \Gamma_{L} \\ \frac{\partial \phi}{\partial n}=h & \text { on } \Gamma_{0}\end{cases}
$$

where if $\Gamma_{0}=\Gamma_{0}^{x} \times \Gamma_{0}^{y}$ with :

$$
\begin{aligned}
& \Gamma_{0}^{x}=\{(x, L), x>0\} \\
& \Gamma_{0}^{y}=\{(0, y), 0<y<L\}
\end{aligned}
$$

the source term $h$ is given by :

$$
\begin{cases}\bullet h=0 \\
\bullet h(y, t)=\left\{\begin{array}{ll}
0 & \text { on } \Gamma_{0}^{x} \\
h(y, t)=A e^{-\frac{\left(t-t_{0}\right)^{2}}{p^{2}}} & \text { for } \frac{L}{2} \leq y \leq L
\end{array} \text { on } \Gamma_{0}^{y}\right.\end{cases}
$$

$x=a$ denotes the abcissa of the artificial boundary that we can choose as we want. The following numerical values are attributed to the data of the problem :

$$
\left\{\begin{array}{l}
g=10 \mathrm{~m} / \mathrm{s}^{2} \\
L=4000 \mathrm{~m} \\
p=11,1408 \mathrm{~s} \\
t_{0}=35 \mathrm{~s} \\
A=0.169
\end{array}\right.
$$

This data gives, for the low frequency waves, the following propagation velocity :

$$
V=\sqrt{g L}=200 \mathrm{~m} / \mathrm{s}
$$

The number $a$ has been chosen in order that the characteristic frequency of the source be :

$$
f_{0}=\frac{1}{\pi a}=\frac{1}{t_{0}}=\frac{1}{35}=0.28610^{-1}
$$


which corresponds to a characteristic wave length

$$
\lambda=V t_{0}=35 \times 200=7000 \mathrm{~m}
$$

The constant $A$ has been computed in order that

$$
\int_{\Gamma_{0}} h d \sigma=1
$$

The rectangular domain $\Omega$ has been discretized with a uniform mesh with rectangles of respective sizes $\Delta x$ in the $x$-direction and $\Delta y$ in the $y$-direction. The triangulation of $\Omega$ is obtained by adding to this mesh the diagonals of the rectangles as indicated on figure 4.1. We then use classical P1 finite elements.

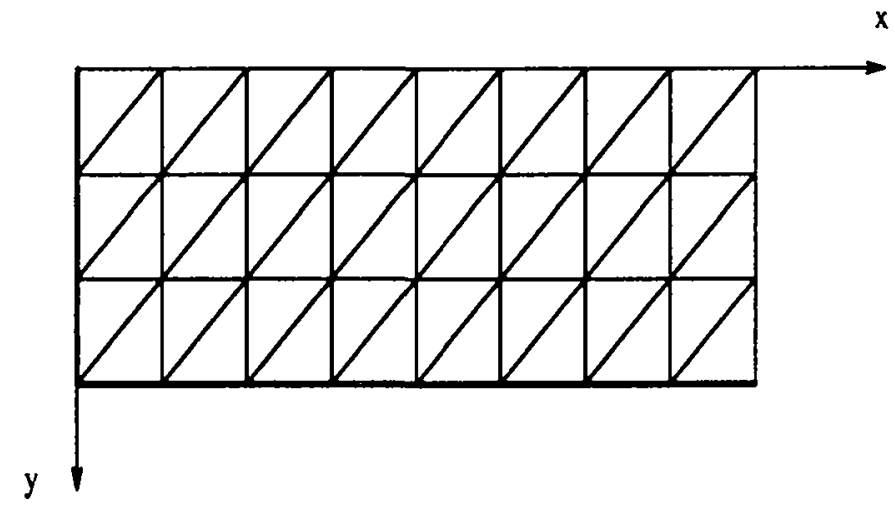

Figure 4.1: The spacial mesh.

For the numerical results we have attributed to the step sizes $\Delta x$ and $\Delta y$ and to the time step $\Delta t$ the following values :

$$
\left\{\begin{array}{l}
\Delta x=\Delta y=500 m \\
\Delta t=2 s
\end{array}\right.
$$

which corresponds approximatively to 12 points per wavelength (and exactely 9 points in depth) and to 35 time steps during the period $2 t_{0}$ where the source is active. Numerical tests of convergence have proven that this was sufficient to get a very good accuracy in the results.

Of course our goal here is to compare the solution calculated with our absorbing boundary condition with the "exact" solution.

In order to make this comparison we shall observe the propagation of the wave generated by our source for a duration of $6 \mathrm{~mm}=360 \mathrm{~s}$ (while the source is cut after $2 t_{0}=70 \mathrm{~s}$ ). With a velocity of $200 \mathrm{~m} / \mathrm{s}$ the corresponding wave travels along a distance approximatively equal to $72.000 \mathrm{~m}=72 \mathrm{~km}$. We have performed our calculations with two values for the abcissa $a$ of our boundary conditions: 


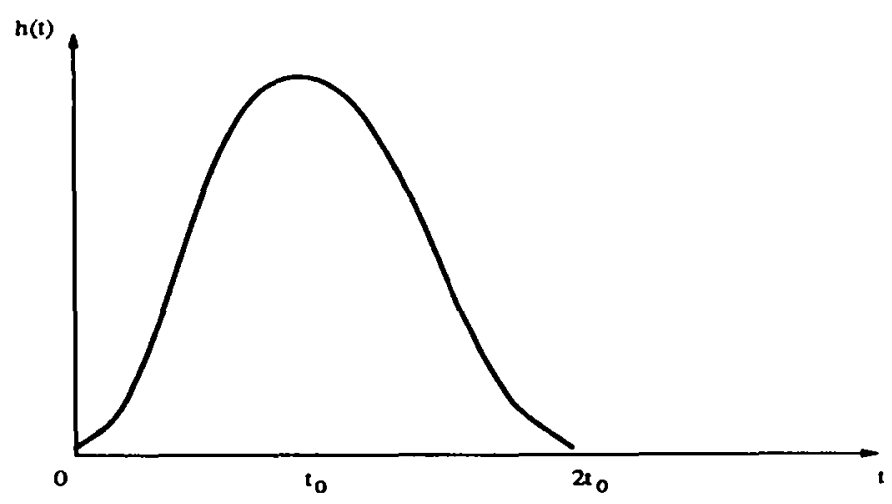

Figure 4.2 : The source function.

$$
\left\{\begin{array}{l}
a=32500 \mathrm{~m} \\
a=65000 \mathrm{~m}
\end{array}\right.
$$

The zone of observation is of course the smaller domain : $\{0 \leq x \leq 32500 \mathrm{~m}\}$ (see Figure 4.3).

During 360s, the solution computed with $a=65000 \mathrm{~m}$ can be considered as the exact solution since the possible reflection by the artificial boundary cannot affect the solution in our zone of observation. Contrarily, we can measure the effect of our absorbing boundary condition with $a=32500 \mathrm{~m}$ since the wave reaches the boundary after approximatively 160 seconds of simulation.

Let us now comment our numerical results. We have tested two boundary conditions :

(i) the condition of order $0(C 0)$, corresponding to the approximation of $f(\omega)$ by :

$$
f_{0}(\omega)=1
$$

(ii) the condition of order $1(C 1)$, corresponding to the approximation of $f(\omega)$ by his first diagonal Padé approximant:

$$
f_{1}(\omega)=1-\frac{b \omega^{2}}{1-a \omega^{2}} ; \quad a=\frac{1}{6}, \quad b=\frac{11}{60} .
$$

In case (ii) the wave dispersion is taken in account and we have to deal with one auxiliary unknown function. 


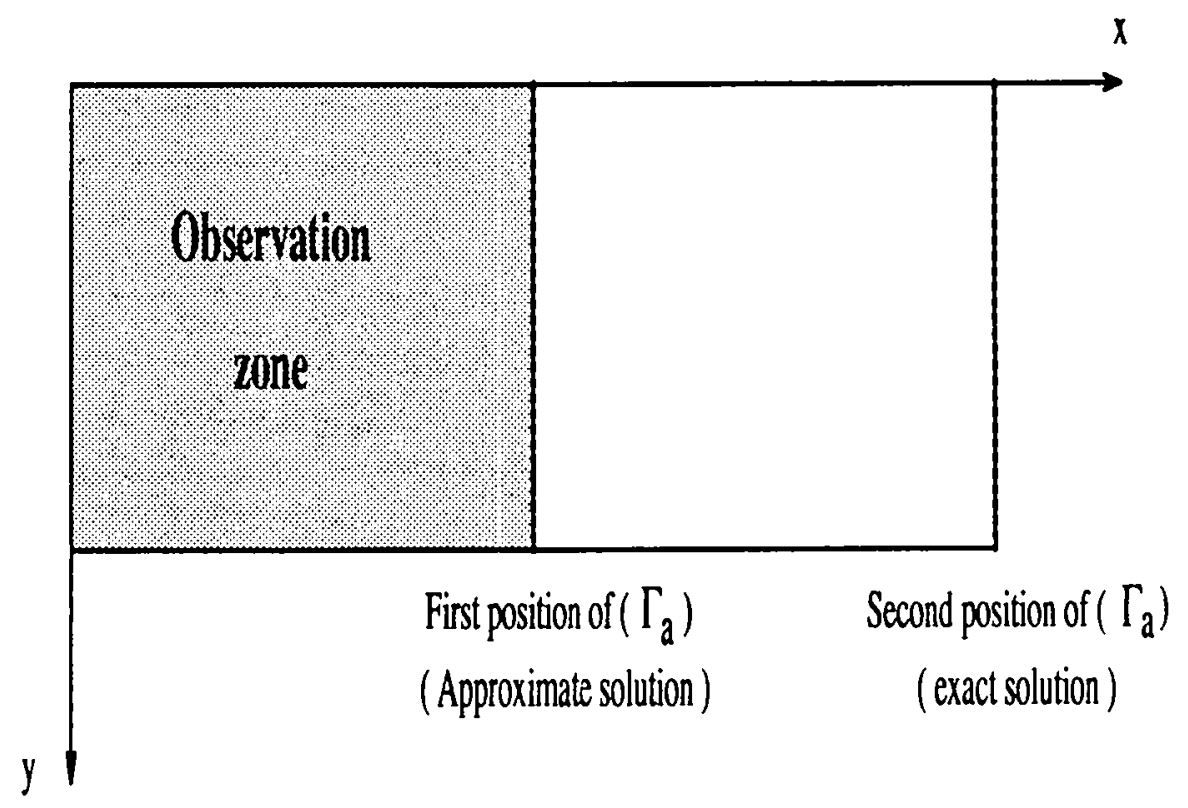

Figure 4.3 : Description of the numerical experiments.

In figures 4.4 to 4.7 we compare the exact solution to the two approximate one (computed with the condition $(C 0)$ and $(C 1))$ by representing the evolution in time of the surface of the water (i.e. the evolution of the function) :

$$
y=\eta(x, t)=\frac{1}{g} \frac{\partial \phi}{\partial t}(x, 0, t) .
$$

Each curve correspond to a snapshot of the solution at a given time and the propagation of the wave is illustrated by the superposition of these curves for equally spaced instants. Of course, on figure 4.4 no significant difference appears as the waves have not yet reached the artificial boundary;but on figures 4.5 to 4.7 we can see that the curves obtained by the condition $(C 0)$ are more different from the exact one than the one obtained by the condition $(C 1)$,especially for later times.

To be able to visualize the effect of the boundary conditions, we have to compare the difference between the exact solution and the $(C 0)$ solution to the difference between the exact solution and the $(C 1)$ solution. This difference could be expected as a travelling back reflected wave.

In figures 4.8 to 4.11 we compare the reflected waves due respectively to the boundary conditions $(C 0)$ and $(C 1)$ from $t=160 \mathrm{~s}$ to $t=380 \mathrm{~s}$. Of course, we have amplified these reflected waves (note the change of scale between figure 4.7 and figure 4.8). We can see that for condition $(C 0)$ the reflected wave becomes visible at $t=160 \mathrm{~s}$ while we have to wait until 220s to distinguish the reflection due to the condition $(C 1)$. At later times 
(figures 4.9 to 4.11 ) we see that the amplitude of the reflected wave by the condition $(C 0)$ is much stronger than the one corresponding to the condition $(C 1)$. In conclusion our results emphasize the interest, even the importance, of taking into account the wave dispersion in the construction of the absorbing boundary condition. 

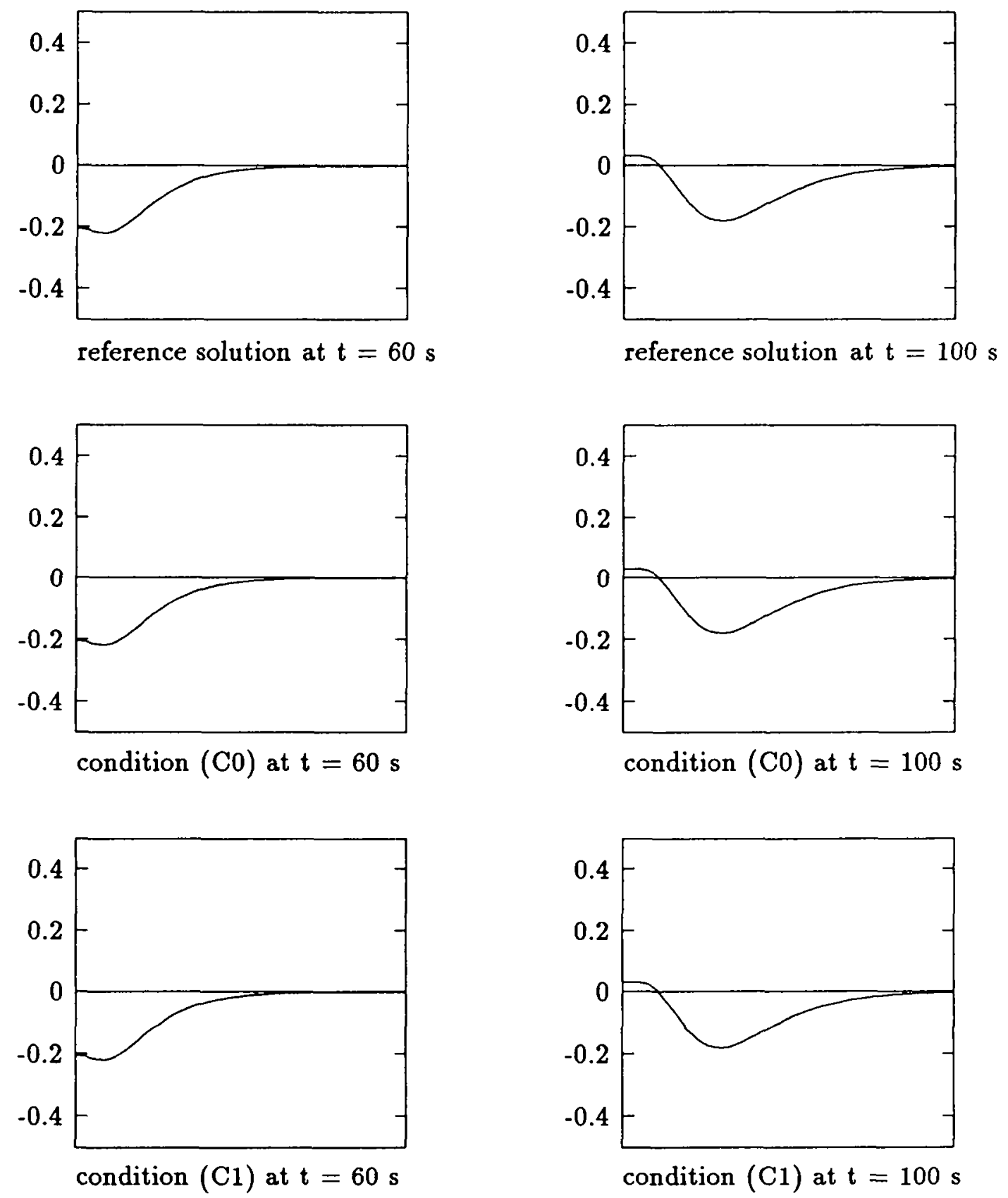

Figure 4.4 Comparison of waves 

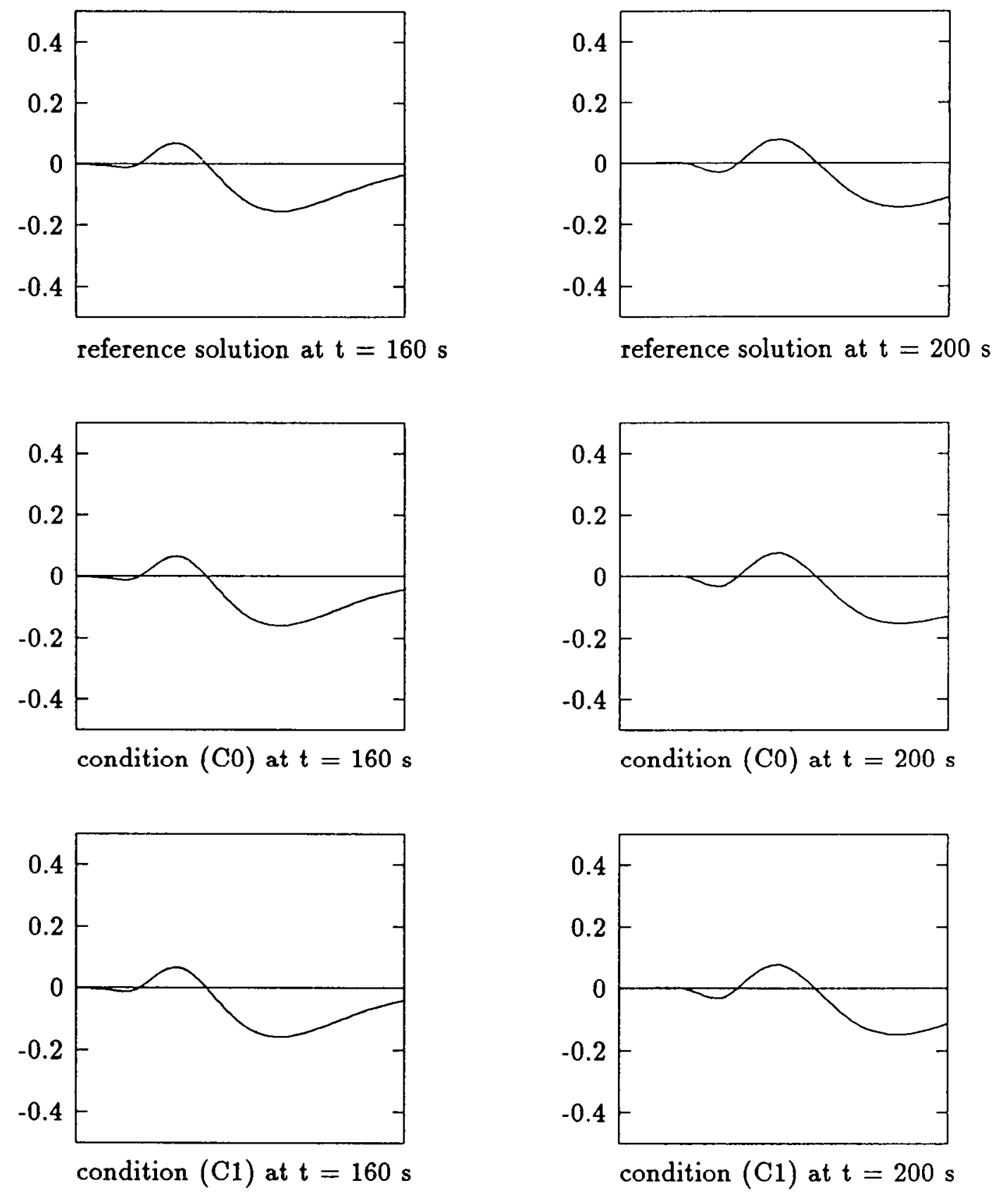

Figure 4.5 Comparison of waves 


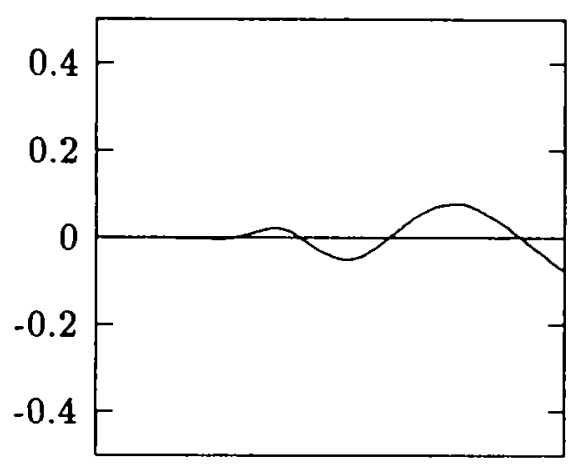

reference solution at $\mathrm{t}=260 \mathrm{~s}$
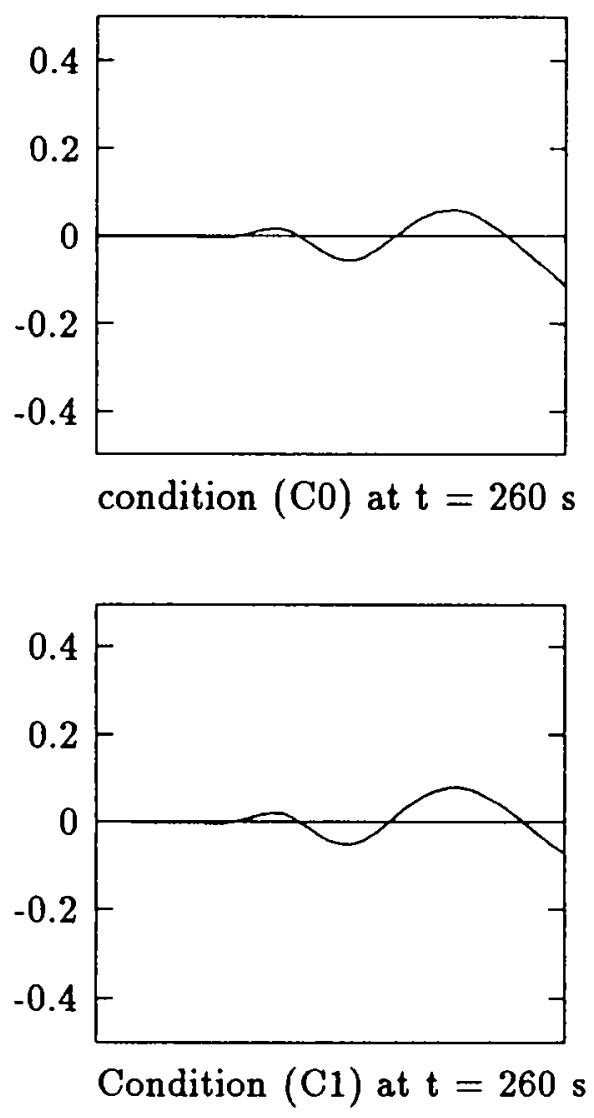
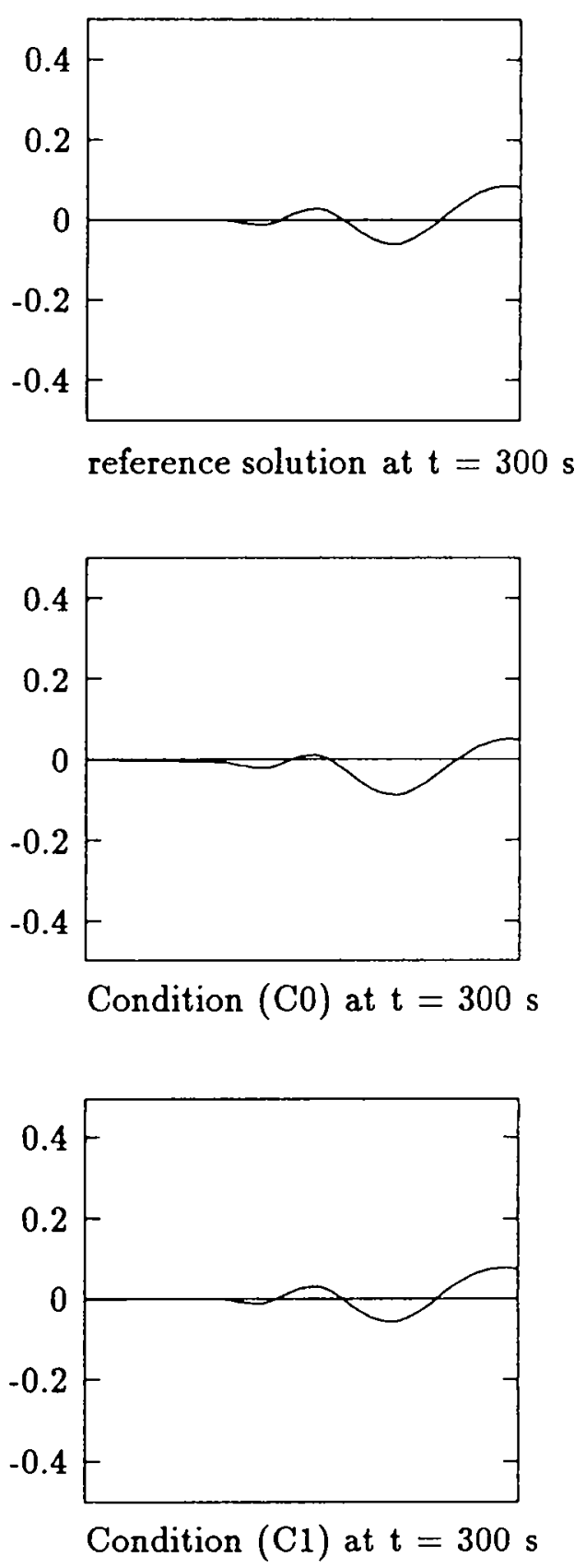


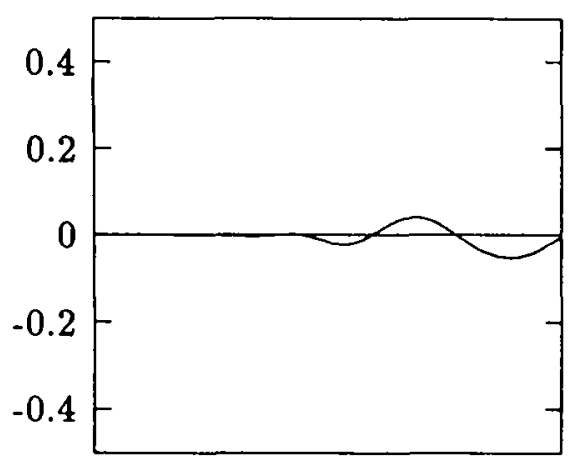

reference solution at $t=340 \mathrm{~s}$

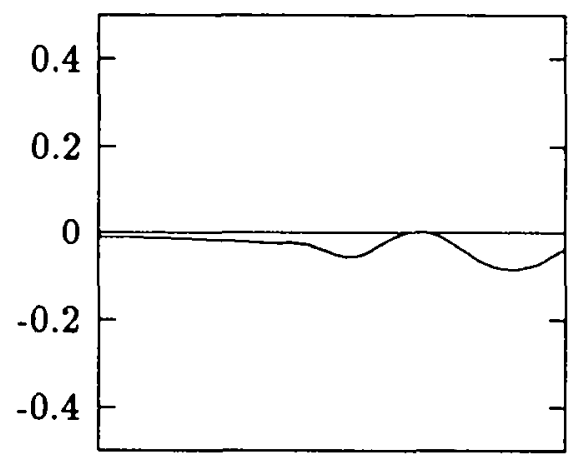

Condition $(\mathrm{C} 0)$ at $\mathrm{t}=340 \mathrm{~s}$

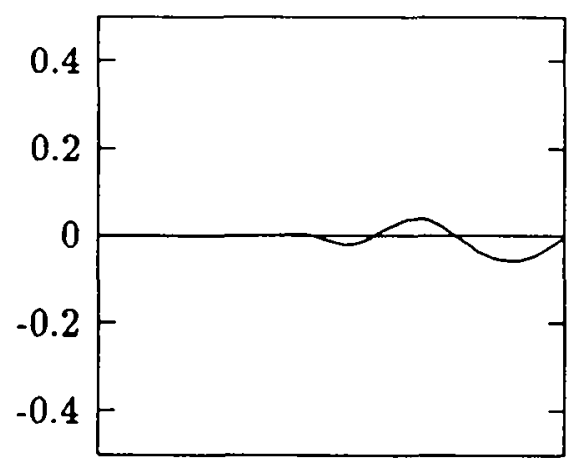

Condition (C1) at $t=340 \mathrm{~s}$

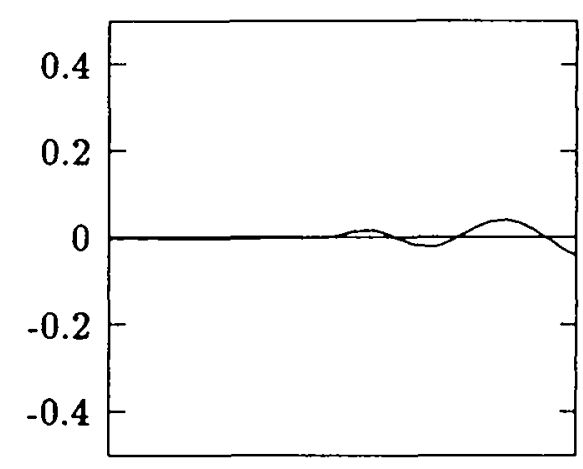

reference solution at $\mathrm{t}=380 \mathrm{~s}$
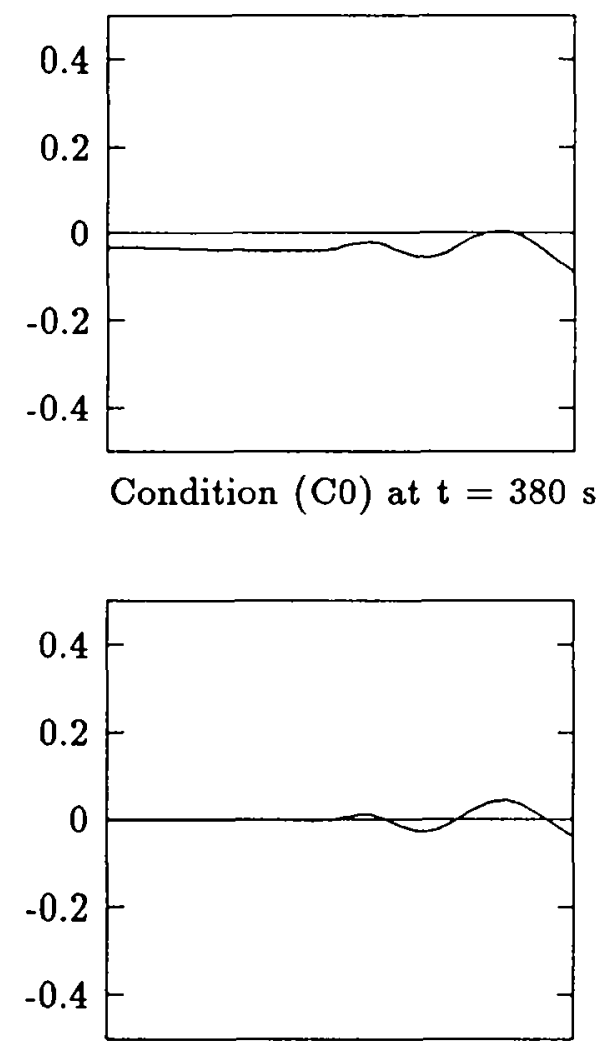

Condition (C1) at $t=380 \mathrm{~s}$

Figure 4.7 Comparison of waves 

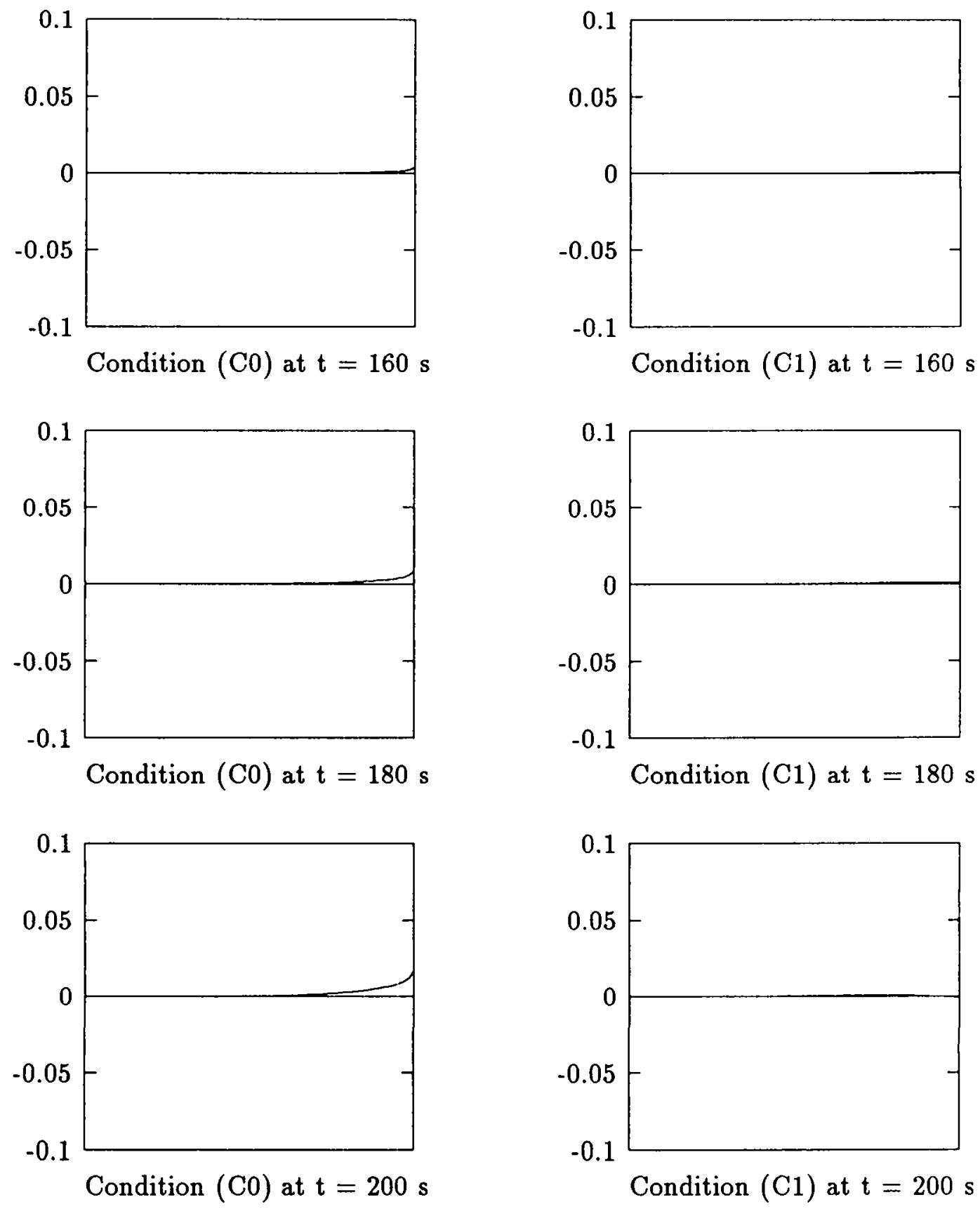

Figure 4.8 Comparison of reflected waves 

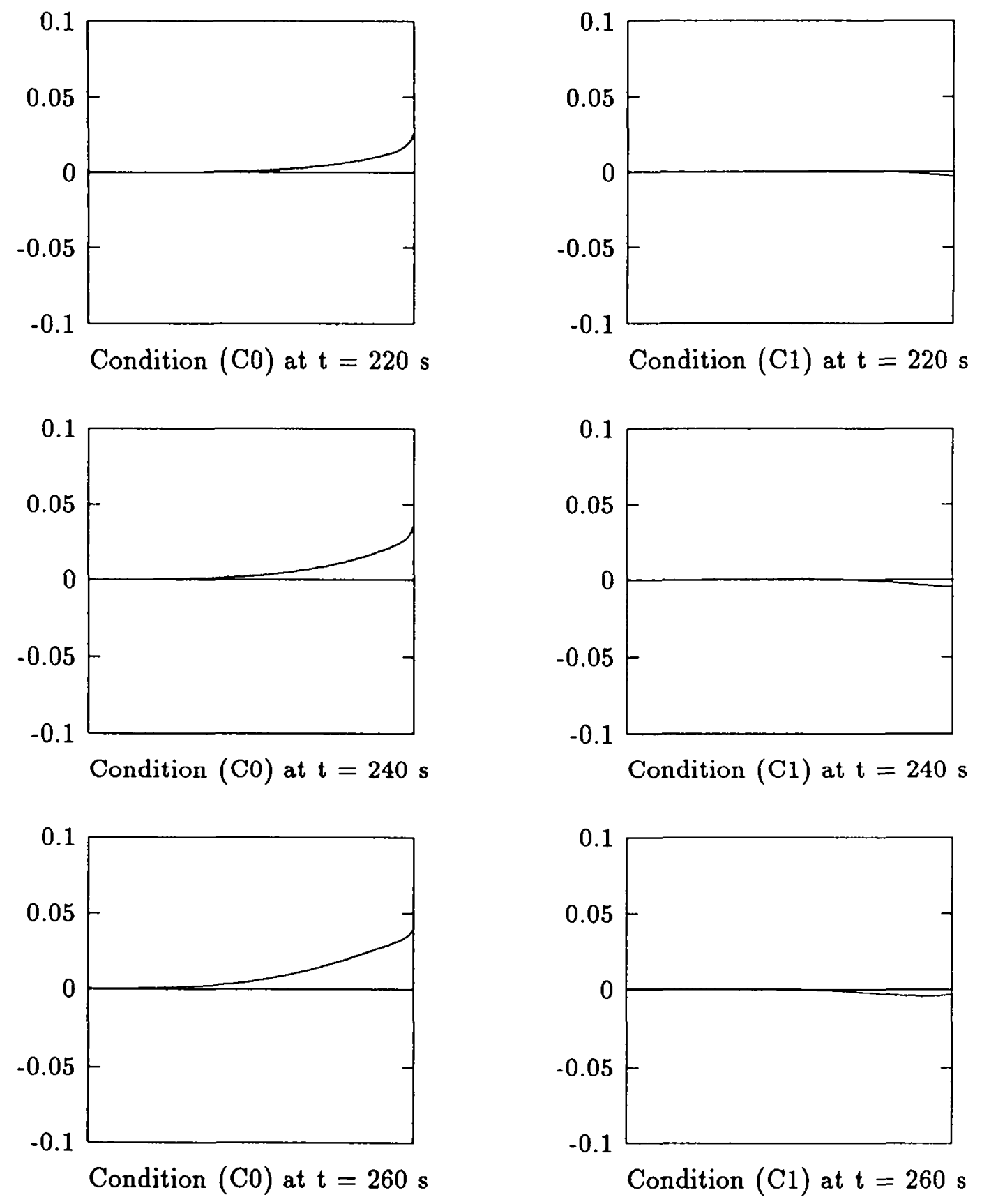

Figure 4.9 Comparison of reflected waves 

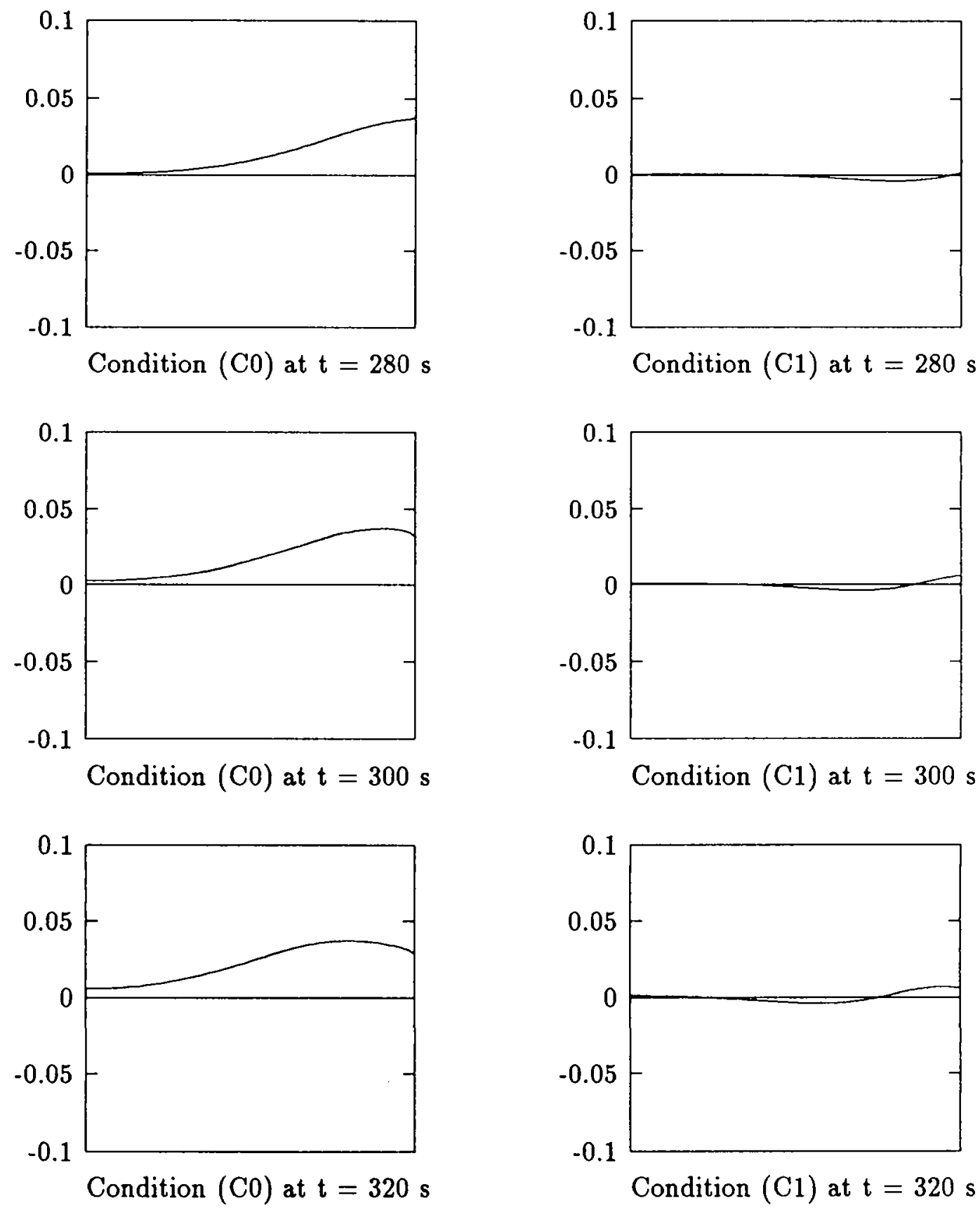

Figure 4.10 Comparison of reflected waves 

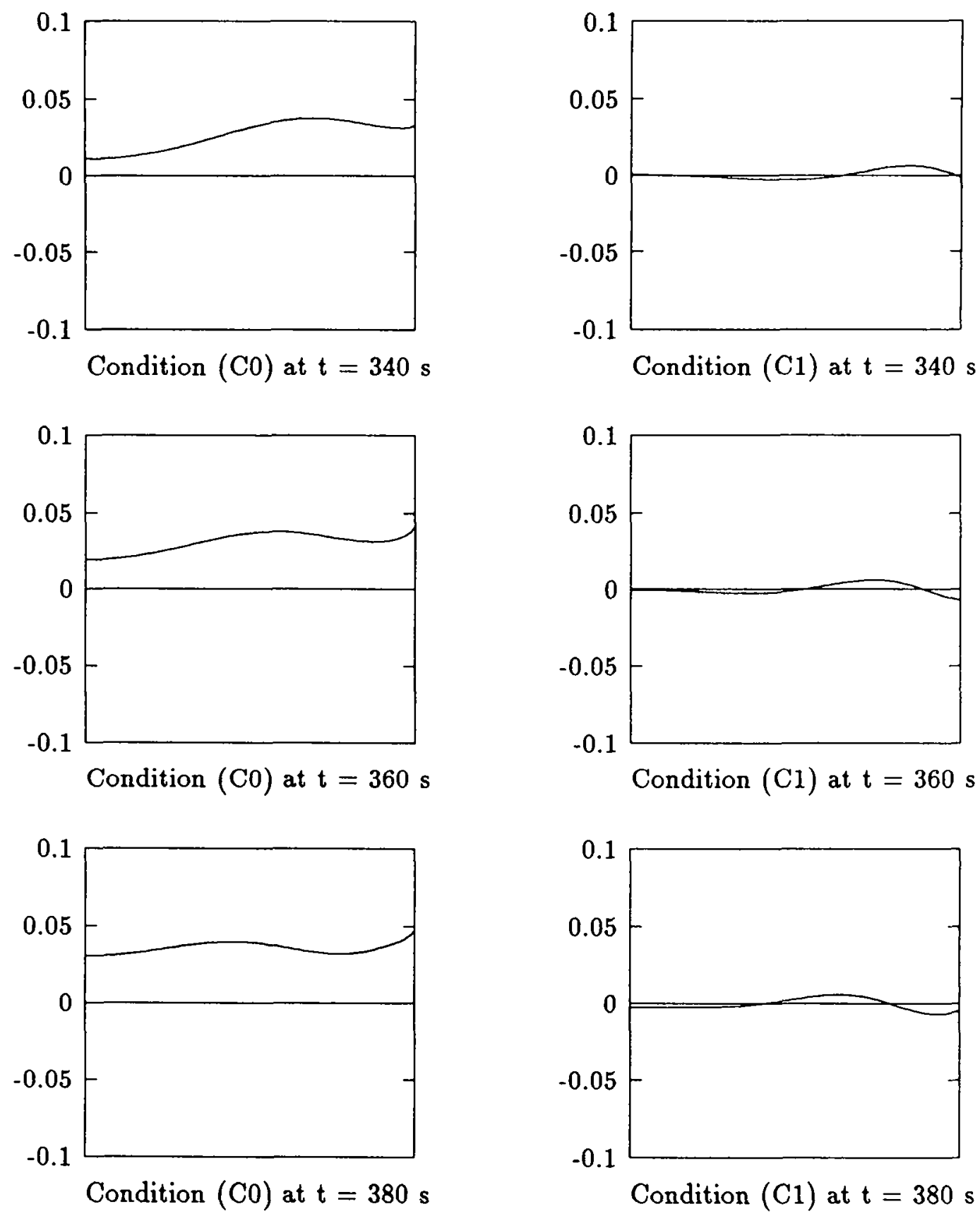

Figure 4.11 Comparison of reflected waves 


\section{References}

[1] R.A. ADAMS, Sobolev spaces, Academic Press (1975).

[2] A. BAMBERGER, B. ENGQUIST, L. HALPERN, P. JOLY, Higher order paraxial approximations to the wave equation, SIAM J. on Appl. Math., Vol. 48, No. 1, pp. 129-154,(1988).

[3] A. BAMBERGER, P. JOLY, J.E. ROBERTS, Second order absorbing boundary conditions for the wave equation : a solution for the corner problem, Rapport INRIA No. 644, to appear in SIAM J. on Appl. Math.

[4] A. BAMBERGER, P. JOLY, J.E. ROBERTS, B. CHALINDAR, J.L. TERON , Absorbing boundary conditions for Rayleigh waves, SIAM J. on Scient. Stat. Computing, Vol. 9, No. 6, 1989.

[5] A. BAYLISS, E. TURKEL, Radiation boundary conditions for wave like equations, Comm. Pure Appl. Math., 33, 707-725 (1980).

[6] J.T. BEALE, Eigenfunction expansions for objects floating in an open sea, Comm. Pure Appl. Math., Vol. 30, No. 3, 1977, pp. 283-313.

[7] A. BENDALI, L. HALPERN, Conditions aux limites absorbantes pour le système de Maxwell dans le vide en dimension 3, Comptes Rendus Acad. Sc. Paris, Tome 307, Série 1 No. 20 (1988).

[8] B. CHALINDAR, Conditions aux limites absorbantes appliquées à des problèmes hyperboliques intervenant en sismique, Thèse, Université de St Etienne, Juin 1989.

[9] G. COHEN, P. JOLY, Fourth order finite difference schemes for the acoustic wave equation, Rapport INRIA to appear, submitted to SIAM J. on Num. Anal.

[10] B. COCKBURN, Resolution of Maxwell's equations in polarizable media at radio and lower frequencies, SIAM J. on Scient. and Stat. Comp., 6, No. 4, pp. 843-852 (1985).

[11] B. COCKBURN, P. JOLY , Maxwell's equations in polarisable media, SIAM J. on Math. Anal., Vol. 19, No. 6, pp. 1372-1390 (1988).

[12] B. ENGQUIST, A. MAJDA, Absorbing boundary conditions for the numerical simulation of waves, Math. Comp., vol. 31, pp. 629-651, July 1977.

[13] B. ENGQUIST, A. MAJDA , Radiation boundary conditions for acoustic and elastic wave calculations, Comm. Pure Appl. Math., 32, pp. 313-357 (1979). 
[14] R.M. GARIPOV, On the linear theory of gravity waves : the theorem of existence and uniqueness, Arch. Rational Mech. Anal., Vol. 24, pp. 352-362 (1966).

[15] T. HAGSTRÖM , Asymptotic expansions and boundary conditions for time dependent problems, SIAM J. on Numer. Anal., Vol. 23, No. 5, (1986).

[16] T. HAGSTRÖM , H.B. KELLER, Exact boundary conditions at an artificial boundary for partial differential equations in cylinders, SIAM J. on Math. Anal., 17, pp. 322-341 (1986).

[17] K. HAMDACHE , Forward speed motions of a submerged body. The Cauchy problem, Math. Methods Appl. Sci., 6, No. 3, 371-392 (1984).

[18] L. HALPERN , Artificial boundary conditions for the linear advection diffusion equation, Math. Comp. 46, 174, 425-438 (1986).

[19] L. HALPERN, M. SCHATZMAN , Conditions aux limites artificielles pour les équations de Navier-Stokes incompressibles, C.R. Acad. Sci. Paris, Série I Math., 304, No. 3, 83-86 (1987).

[20] R. HERSCH , Mixed problems in several variables, J. Math. Mech., 12, pp. 317-334 (1963).

[21] R. HIGDON , Initial boundary value problems for linear hyperbolic systems, S.I.A.M. Review, Vol. 28, No. 2, (1986).

[22] P. JOLY, B. MERCIER, Une nouvelle condition transparente d'ordre 2 pour les équations de Maxwell en dimension 3, Rapport INRIA No. 1047 (1989).

[23] P. JOLY, Pseudo transparent boundary conditions for the diffusion equation, Part I, Math. Meth. in Appl. Sciences, Vol. 11, 725-758 (1989).

[24] H.O. KREISS , Initial boundary value problems for hyperbolic systems, Comm. Pure and Appl. Math., 23, pp. 277-298 (1970).

[25] M. LENOIR, A. TOUNSI, The localized finite element method to the two-dimensional see keeping problem, SIAM J. on Num. Anal., Vol. 28, No. 4, Août 1988.

[26] M. LENOIR, M. VERRIERE , Computation of transient gravity waves, 4 th Workshop on water waves and floating bodies, Oytsee, Norvège (7-10 Mai 1989).

[27] J.L. LIONS, E. MAGENES , Problèmes aux limites non homogènes et applications, Dunod (1968). 
[28] D.H. RUDY, J. STRIKWERKA, A non reflecting outflow boundary condition for subsonic Navier-Stokes calculations, J. of Comp. Physics, 36, 55-70 (1980).

[29] J.J. STOCKER, Waterwaves, Interscience.

[30] L.N. TREFETHEN, L. HALPERN, Well posedness of one way wave equations and absorbing boundary conditions, Math. Comp., 47, pp. 437-459 (1986).

[31] M. VERRIERE, Calcul numérique de champ de vagues linéaires en régime transitoire en présence d'un obstacle tridimensionnel, Thèse, ENSTA, Septembre 1989.

[32] B.R. WAINBERG, Hypoelliptic equations in the whole space and the principle of limiting absorption, Soviet Math. Dokl., 5, pp. 321-324, 1964.

[33] C.H. WILCOX, Scattering theory for the d'Alembert equation in exterior domains, Lecture Notes in mathematics, No. 442, Springer-Verlag. 
ISSN $0249-6399$ 